\title{
Argyres-Douglas theories and Liouville irregular states
}

\section{Takahiro Nishinaka and Takahiro Uetoko}

Department of Physical Sciences, College of Science and Engineering, Ritsumeikan University, Shiga 525-8577, Japan

E-mail: nishinak@fc.ritsumei.ac.jp, rp0019fr@ed.ritsumei.ac.jp

ABSTRACT: We study irregular states of rank-two and three in Liouville theory, based on an ansatz proposed by D. Gaiotto and J. Teschner. Using these irregular states, we evaluate asymptotic expansions of irregular conformal blocks corresponding to the partition functions of $\left(A_{1}, A_{3}\right)$ and $\left(A_{1}, D_{4}\right)$ Argyres-Douglas theories for general $\Omega$-background parameters. In the limit of vanishing Liouville charge, our result reproduces strong coupling expansions of the partition functions recently obtained via the Painlevé/gauge correspondence. This suggests that the irregular conformal block for one irregular singularity of rank 3 on sphere is also related to Painlevé II. We also find that our partition functions are invariant under the action of the Weyl group of flavor symmetries once four and twodimensional parameters are correctly identified. We finally propose a generalization of this parameter identification to general irregular states of integer rank.

KEywords: Conformal Field Theory, Extended Supersymmetry, Supersymmetric Gauge Theory

ArXiv EPrint: 1905.03795 


\section{Contents}

1 Introduction 1

$2 \quad$ AD theories of class $\mathcal{S}$ and AGT correspondence 3

2.1 AD theories of class $\mathcal{S}$

2.2 Irregular states and generalized AGT correspondence 5

$\begin{array}{lll}3 & \text { Partition functions from irregular states } & 7\end{array}$

$3.1\left(A_{1}, D_{4}\right)$ theory from rank-2 irregular state 8

3.1.1 Ansatz in terms of generalized descendants 8

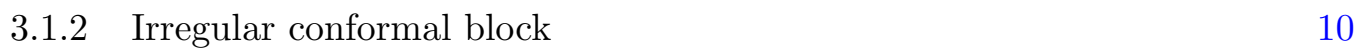

$3.2\left(A_{1}, A_{3}\right)$ theory from rank-3 irregular state $\quad 12$

$\begin{array}{lll}3.2 .1 & \text { Irregular conformal block } & 13\end{array}$

4 Enhanced flavor symmetries $\quad 16$

$\begin{array}{lll}\text { 4.1 Weyl group of the flavor symmetry } & 16\end{array}$

$\begin{array}{lll}4.1 .1 & \left(A_{1}, D_{4}\right) \text { theory } & 17\end{array}$

$\begin{array}{lll}4.1 .2 & \left(A_{1}, A_{3}\right) \text { theory } & 18\end{array}$

$\begin{array}{lll}4.2 & \text { Conjectural dictionary between } 4 \mathrm{~d} \text { and } 2 \mathrm{~d} \text { parameters } & 18\end{array}$

4.2.1 $\left(A_{1}, D_{4}\right)$ partition function 20

$\begin{array}{lll}4.2 .2 & \left(A_{1}, A_{3}\right) \text { partition function } & 20\end{array}$

5 Conclusions and discussions $\quad 21$

A Computations of generalized descendants $\quad 22$

A.1 Rank-two state $\left|I^{(2)}(\alpha, \boldsymbol{c} ; \boldsymbol{\beta})\right\rangle \quad 22$

A.2 Rank-three state $\left|I^{(3)}(\alpha, \boldsymbol{c} ; \boldsymbol{\beta})\right\rangle \quad 22$

B More coefficients for the $\left(A_{1}, D_{4}\right)$ partition function 23

\section{Introduction}

Argyres-Douglas (AD) theories are an important series of strongly coupled $\mathcal{N}=2$ superconformal field theories (SCFTs) in four dimensions [1-3]. Although they are often obtained as IR SCFTs of RG-flows from $\mathcal{N}=2$ gauge theories, their physics is still to be understood. One reason for this is that, since the $\mathrm{U}(1)_{R}$ symmetry of the $\mathrm{AD}$ theories is accidental in the RG-flows, it is hard to read off the IR physics from its UV description. ${ }^{1}$ The accidental $\mathrm{U}(1)_{R}$ symmetry particularly makes it difficult to compute the partition functions of the $\mathrm{AD}$ theories via supersymmetric localization. Therefore, until recently, the partition functions of $\mathrm{AD}$ theories were only partially computed for massless cases via the holomorphic anomaly equations [14].

\footnotetext{
${ }^{1}$ For recent developments in the study of $\mathcal{N}=1$ Lagrangian theories that flow into the $\mathrm{AD}$ theories, see $[4-13]$.
} 
However, there has recently been remarkable progress in the study of the partition functions of $\mathrm{AD}$ theories. In particular, the authors of [15] have evaluated strong coupling expansions of the Nekrasov partition functions of the $\left(A_{1}, A_{2}\right),\left(A_{1}, A_{3}\right)$ and $\left(A_{1}, D_{4}\right) \mathrm{AD}$ theories via a remarkable relation to the tau-functions of Painlevé equations, inspired by [16] and subsequent works [17-20]. ${ }^{2}$ After these works, the author of [24-26] has nicely shown that these strong coupling expansions can be reproduced from irregular conformal blocks of Virasoro algebra. ${ }^{3}$ The emergence of the two-dimensional conformal field theory is naturally understood via the AGT correspondence [28, 29] and its generalization [30, 31] that relate the Nekrasov partition functions of $4 \mathrm{~d} \mathcal{N}=2$ theories to conformal blocks of two-dimensional Liouville theory. Indeed, an attempt to compute the partition function of AD theories as Liouville irregular conformal blocks was partially studied in $[30,31] .{ }^{4}$ Finally, nice matrix model descriptions of this relation between $\mathrm{AD}$ theories and Painlevé equations have been studied in [37-39]. ${ }^{5}$

In this paper, we extend the irregular conformal block approach to various directions, focusing on the $\left(A_{1}, A_{3}\right)$ and $\left(A_{1}, D_{4}\right)$ AD theories. First, we compute the irregular conformal blocks for these two AD theories using an ansatz proposed in [31]. This ansatz is based on the construction of an irregular state in a colliding limit of regular vertex operators, and implies that a rank- $n$ irregular state is expanded in terms of "generalized descendants" of a rank- $(n-1)$ irregular state. Here, generalized decendants are descendants in the sense of Virasoro generators and derivatives with respect to parameters characterizing irregular states. Since this construction of irregular conformal blocks is different from the one used in [24-26], it is non-trivial whether it leads to a consistent result. In this paper, we explicitly show that the ansatz mentioned above gives a consistent result, and moreover we demonstrate that the asymptotic expansion of the ansatz proposed in [31] precisely corresponds to the strong coupling expansion of partition functions obtained in [15].

Second, we reproduce the partition function of the $\left(A_{1}, A_{3}\right)$ theory from the rank-three irregular state of the Liouville theory. This partition function was reproduced in [26] as the irregular conformal block with an irregular singular point of rank $\frac{3}{2}$ and a regular singular point. On the other hand, from the class $\mathcal{S}$ construction of the $\left(A_{1}, A_{3}\right)$ theory, the same partition function is expected to be obtained as the one-point function of an irregular vertex operator of rank 3 . We explicitly show that this is indeed the case.

Finally, we evaluate these irregular conformal blocks with non-vanishing Liouville charge $Q$ turned on. ${ }^{6}$ Since the Painlevé tau-functions are related to the case of $Q=0$, earlier works focused on the vanishing Liouville charge. On the other hand, in relation to the $4 \mathrm{~d}$ physics, it is important to evaluate the irregular conformal blocks for non-vanishing $Q$. Indeed, according to the AGT correspondence, the Liouville charge $Q$ corresponds to $\left(\epsilon_{1}+\epsilon_{2}\right) / \sqrt{\epsilon_{1} \epsilon_{2}}$ in four dimensions, where $\epsilon_{1}$ and $\epsilon_{2}$ are two $\Omega$-background parameters [67].

\footnotetext{
${ }^{2}$ Another important progress in the study of the partition functions of $\mathrm{AD}$ theories has been made through the E-string theory on $T^{2}$ [21] and quantum periods in the Nekrasov-Shatashvili limit [22, 23].

${ }^{3}$ See also [27] for closely related results related to asymptotically free gauge theories.

${ }^{4}$ For more works on Liouville irregular states, see [29, 32-36].

${ }^{5}$ Matrix model descriptions of the partition functions of $4 \mathrm{~d} \mathcal{N}=2$ theories in the context of the AGT correspondence were studied in various papers. See for example [40-66].

${ }^{6}$ Our convention for the Liouville charge is such that the Virasoro central charge is given by $c=1+6 Q^{2}$.
} 
Therefore, the non-vanishing $Q$ leads to the partition function of AD theories with the most general $\Omega$-background turned on. We particularly show that the four-dimensional flavor symmetry implies that there exists an $\mathcal{O}(Q)$ correction to the relation between $4 \mathrm{~d}$ and $2 \mathrm{~d}$ parameters. We interpret this $\mathcal{O}(Q)$ correction in terms of the colliding limit of regular vertex operators, which leads us to a conjecture for a general irregular state of integer rank.

The organization of the paper is the following. In section 2, we briefly review the class $\mathcal{S}$ construction of AD theories and the generalized AGT correspondence. In section 3, we evaluate the Liouville irregular states of rank-two and three, using the ansatz proposed in [31]. We then use these irregular states to compute the irregular conformal blocks corresponding to the partition functions of $\left(A_{1}, D_{4}\right)$ and $\left(A_{1}, A_{3}\right)$ theories for a general $\Omega$-background parameters $\epsilon_{1}$ and $\epsilon_{2}$. In section 4, we show that the partition functions of the $\left(A_{1}, D_{4}\right)$ and $\left(A_{1}, A_{3}\right)$ theories evaluated in section 3 are invariant under the action of the Weyl group of the enhanced flavor symmetry, once parameters in four and two dimensions are appropriate identified. We also conjecture a general identification of the Liouville momentum of irregular states with a $4 \mathrm{~d}$ mass parameter. In section 5 , we conclude with a comment on the symmetry of the partition functions under $\left(\epsilon_{1}, \epsilon_{2}\right) \rightarrow\left(-\epsilon_{1},-\epsilon_{2}\right)$.

\section{$2 \quad$ AD theories of class $\mathcal{S}$ and AGT correspondence}

In this section, we briefly review the $\left(A_{1}, A_{3}\right)$ and $\left(A_{1}, D_{4}\right)$ theories as well as the generalized AGT correspondence that relates the partition functions of AD theories to irregular conformal blocks of Liouville theory in two dimensions.

\subsection{AD theories of class $\mathcal{S}$}

The AD theories are a series of strongly coupled $4 \mathrm{~d} \mathcal{N}=2$ SCFTs with Coulomb branch operators of fractional dimensions. While the simplest AD theories were originally discovered as IR SCFTs at special points on the Coulomb branch of asymptotically free gauge theories [1-3], their infinite generalizations were constructed by compactifying $6 \mathrm{~d}(2,0)$ SCFTs with gauge algebra $\mathfrak{g}$ on a punctured Riemann surface $\mathcal{C}[30,68] .{ }^{7}$ In this paper, we focus on $\mathfrak{g}=A_{1}$. Then the resulting $\mathrm{AD}$ theories are $\left(A_{1}, A_{N}\right)$ and $\left(A_{1}, D_{N}\right)$ theories, depending on the number and types of the punctures on $\mathcal{C}$. In the rest of this sub-section, we denote by $\mathcal{T}_{\mathcal{C}}$ the $4 \mathrm{~d} \mathcal{N}=2$ theory obtained by compactifying $6 \mathrm{~d}(2,0) A_{1}$ theory on $\mathcal{C}$.

As mentioned above, the $4 \mathrm{~d}$ theory $\mathcal{T}_{\mathcal{C}}$ depends on the number and types of the punctures on $\mathcal{C}$. Possible punctures on $\mathcal{C}$ are classified into two types; regular and irregular punctures. To describe these two types, let us briefly review how the Coulomb branch moduli space of the $4 \mathrm{~d}$ theory $\mathcal{T}_{\mathcal{C}}$ is related to the Hitchin system on $\mathcal{C}$. Since we focus on the case of $\mathfrak{g}=A_{1}$, we consider the $A_{1}$ Hitchin system on $\mathcal{C}$, which involves an $\mathrm{SU}(2)$ connection $A$ on $\mathcal{C}$ and an $\mathfrak{s u}(2)$-valued 1-form $\varphi d z+\bar{\varphi} d \bar{z}$ on $\mathcal{C}$. The pair $(A, \varphi)$ is required to satisfy a set of differential equations called "Hitchin's equations." Since the Hitchin's equations are differential equations, we need to specify the boundary conditions of $A$ and

\footnotetext{
${ }^{7}$ See also [69] for the construction of $\mathrm{AD}$ theories via type II string theory on Calabi-Yau singularities. Indeed, the $\mathrm{AD}$ theories we discuss in this paper, $\left(A_{1}, A_{3}\right)$ and $\left(A_{1}, D_{4}\right)$, were first constructed in this reference.
} 
$\varphi$ at each puncture on $\mathcal{C}$. We call a puncture a "regular puncture" if $\varphi$ has a simple pole there, while a puncture is called an "irregular puncture" if the singularity of $\varphi$ is not a simple pole. Suppose that an irregular puncture is located at $z=0$. When $\varphi$ behaves as $\varphi \sim 1 / z^{n+1}$ around it, we call $n$ the "rank" of the irregular puncture. While the rank could be an integer or a half-integer for $\mathfrak{g}=A_{1}$, we focus on integer ranks in this paper. The Hitchin moduli space is then defined as the space of solutions to Hitchin's equations (modulo gauge transformations) with the boundary conditions at punctures kept fixed. The spectral curve of the Hitchin system is defined by

$$
\operatorname{det}(x d z-\varphi)=0 .
$$

A beautiful observation of [70-72] is that this spectral curve is identified with the SeibergWitten (SW) curve of the corresponding $4 \mathrm{~d} \mathcal{N}=2$ theory $\mathcal{T}_{\mathcal{C}}{ }^{8}$ Note that this SW curve, and therefore the $4 \mathrm{~d}$ theory $\mathcal{T}_{\mathcal{C}}$, depend on the number and types of the punctures on $\mathcal{C}$. In particular, the flavor symmetry of $\mathcal{T}_{\mathcal{C}}$ is naturally encoded in the punctures. Indeed, each regular puncture gives rise to a flavor $\mathrm{SU}(2)$ symmetry, while each irregular puncture of integer rank gives rise to a flavor $\mathrm{U}(1)$ symmetry in $\mathcal{T}_{\mathcal{C}} \cdot{ }^{9}$ As discussed in [71, 72], when $\mathcal{C}$ has no irregular puncture, $\mathcal{T}_{\mathcal{C}}$ is always an $\mathcal{N}=2$ SCFT. ${ }^{10}$ On the other hand, when $\mathcal{C}$ has an irregular puncture, the $\mathcal{N}=2$ supersymmetric field theory $\mathcal{T}_{\mathcal{C}}$ is conformal if and only if $\mathcal{C}$ is of genus zero, has one or zero regular puncture, and does not have more than one irregular puncture $[30,68]$.

Let us now describe the $\left(A_{1}, A_{N}\right)$ and $\left(A_{1}, D_{N}\right)$ theories. The $\left(A_{1}, A_{2 n-3}\right)$ theory is a $4 \mathrm{~d} \mathcal{N}=2$ SCFT associated with $\mathcal{C}$ being a sphere with an irregular puncture of rank $n$. On the other hand, the $\left(A_{1}, D_{2 n}\right)$ theory is $4 \mathrm{~d} \mathcal{N}=2$ SCFT associated with $\mathcal{C}$ which is a sphere with a regular puncture and an irregular puncture of rank $n$. These two theories are $\mathrm{AD}$ theories since they have fractional-dimensional Coulomb branch operators. In this paper, we focus on two $\mathrm{AD}$ theories in these series; $\left(A_{1}, A_{3}\right)$ and $\left(A_{1}, D_{4}\right)$. These two $\mathrm{AD}$ theories are special in the sense of flavor symmetry enhancement. Indeed, according to the relation between the flavor symmetry and the punctures on $\mathcal{C}$, the manifest flavor symmetries of $\left(A_{1}, A_{2 n-3}\right)$ and $\left(A_{1}, D_{2 n}\right)$ for an integer $n$ are $\mathrm{U}(1)$ and $\mathrm{SU}(2) \times \mathrm{U}(1)$, respectively. However, for $\left(A_{1}, A_{3}\right)$ and $\left(A_{1}, D_{4}\right)$ theories, these manifest flavor symmetries get enhanced to $\mathrm{SU}(2)$ and $\mathrm{SU}(3)$, respectively. This flavor symmetry enhancement will be important in our discussions in section 4.

For later use, we here write down the SW curves of these two theories. Up to a change of variables, the SW curve $(2.1)$ for the $\left(A_{1}, A_{3}\right)$ theory is written as ${ }^{11}$

$$
x^{2}=\frac{u}{z^{4}}+\frac{m}{z^{5}}+\frac{c}{z^{6}}+\frac{1}{z^{8}},
$$

\footnotetext{
${ }^{8}$ The Seiberg-Witten 1-form is identified with $x d z$.

${ }^{9}$ Irregular punctures of non-integer rank do not give rise to a flavor symmetry. We will not use them in this paper.

${ }^{10}$ To be more precise, $\mathcal{C}$ needs at least three regular punctures for $\mathcal{T}_{\mathcal{C}}$ to be a non-trivial $\mathcal{N}=2 \mathrm{SCFT}$.

${ }^{11}$ We here take the holomorphic coordinate $z$ on $\mathcal{C}$ so that the irregular puncture is located at $z=0$. In the literature, the SW curve of the $\left(A_{1}, A_{3}\right)$ theory is usually written as

$$
x^{2}=z^{4}+c z^{2}+m z+u,
$$

which is related to $(2.3)$ by the change of coordinates $(x, z) \rightarrow\left(-z^{2} x, 1 / z\right)$. Note that this coordinate change preserves the SW 1-form $x d z$.
} 
where $c, m$ and $u$ are coefficients respectively interpreted as a relevant coupling of dimension 2/3, a mass parameter, and the vacuum expectation value (vev) of a Coulomb branch operator of dimension $4 / 3$. On the other hand, for the $\left(A_{1}, D_{4}\right)$ theory, the SW curve is written as ${ }^{12}$

$$
x^{2}=\frac{M^{2}}{z^{2}}+\frac{u}{z^{3}}+\frac{m}{z^{4}}+\frac{c}{z^{5}}+\frac{1}{z^{6}},
$$

where $c$ and $u$ are respectively a relevant coupling of dimension $1 / 2$ and the vev of a Coulomb branch operator of dimension $3 / 2$, while $m$ and $M$ are two mass parameters. See $[30,68]$ for more detail.

\subsection{Irregular states and generalized AGT correspondence}

We here review the generalized AGT correspondence that relates the partition functions of $\mathrm{AD}$ theories to irregular conformal blocks of two-dimensional Liouville theory. Let $\mathcal{T}_{\mathcal{C}}$ be a $4 \mathrm{~d} \mathcal{N}=2$ SCFT associated with a Riemann surface $\mathcal{C}$ with only regular punctures. Then the AGT correspondence [28] states that the Nekrasov partition functions [67] of $\mathcal{T}_{\mathcal{C}}$ to the conformal block of Liouville theory on $\mathcal{C}$. Here, each regular puncture on $\mathcal{C}$ corresponds to the insertion of a Virasoro primary vertex operator $e^{2 \gamma \phi(z)}$ whose Liouville momentum $\gamma$ is fixed by the mass parameter associated with the regular puncture. Therefore, when $\mathcal{C}$ has $\ell$ regular punctures, then the partition function of $\mathcal{T}_{\mathcal{C}}$ is identified with the $\ell$-point conformal block of Liouville theory, i.e.,

$$
Z_{\mathcal{T}_{\mathcal{C}}}^{\epsilon_{1}, \epsilon_{2}}=\mathcal{F}_{\gamma_{1}}{ }^{\gamma_{2}}{ }_{\gamma_{3}} \ldots{ }^{\gamma_{\ell-1}}{ }_{\gamma_{\ell}}
$$

where $\epsilon_{1}$ and $\epsilon_{2}$ are the $\Omega$-background parameters turned on in four dimensions. In this paper, we fix the scale of the l.h.s. by setting $\epsilon_{1} \epsilon_{2}=1$ as in [28]. The ratio of the $\Omega$ background parameters is related to the Liouville charge $Q$ by ${ }^{13}$

$$
Q=b+\frac{1}{b}, \quad b^{2} \equiv \frac{\epsilon_{1}}{\epsilon_{2}} .
$$

It was also proposed in [28] that the $\mathrm{SW}$ curve of $\mathcal{T}_{\mathcal{C}}$ is identified as the Liouville correlator with a stress tensor insertion:

$$
x^{2}=\frac{\left\langle\Delta_{\gamma_{1}}\left|T(z) e^{2 \gamma_{2} \phi} \cdots e^{2 \gamma_{\ell-1} \phi}\right| \Delta_{\gamma_{\ell}}\right\rangle}{\left\langle\Delta_{\gamma_{1}}\left|e^{2 \gamma_{2} \phi} \cdots e^{2 \gamma_{\ell-1} \phi}\right| \Delta_{\gamma_{\ell}}\right\rangle},
$$

where $\left|\Delta_{\gamma}\right\rangle$ is the Virasoro primary state of holomorphic dimension $\Delta_{\gamma} \equiv \gamma(Q-\gamma)$.

A generalization of the AGT correspondence to AD theories has been discussed in $[30,31],{ }^{14}$ in which an irregular puncture of rank $n$ is conjectured to correspond to

\footnotetext{
${ }^{12}$ Again, in the literature, this curve is usually written as

$$
x^{2}=z^{2}+c z+m+\frac{u}{z}+\frac{M^{2}}{z^{2}}
$$

which is related to $(2.5)$ by $(x, z) \rightarrow\left(-z^{2} x, 1 / z\right)$.

${ }^{13}$ Recall here that our convention for $Q$ is such that the Virasoro central charge is given by $c=1+6 Q^{2}$.

${ }^{14}$ See also [29] for the first generalization to class $\mathcal{S}$ theories whose Riemann surface involves irregular punctures.
} 
a particular linear combination, $\left|I^{(n)}\right\rangle$, of a Virasoro primary and its descendants. In this paper, we follow the construction of $\left|I^{(n)}\right\rangle$ proposed in [31]. Namely, the state $\left|I^{(n)}\right\rangle$ is a solution to the following $2 n$ equations involving $(n+1)$ parameters $c_{1}, \cdots, c_{n}$ and $\alpha$ :

$$
L_{k}\left|I^{(n)}\right\rangle= \begin{cases}\Lambda_{k}\left|I^{(n)}\right\rangle & \text { for } \quad n \leq k \leq 2 n \\ \left(\Lambda_{k}+\sum_{\ell=k+1}^{n-1}(\ell-k) c_{\ell} \frac{\partial}{\partial c_{\ell-k}}\right)\left|I^{(n)}\right\rangle & \text { for } \quad 1 \leq k \leq n-1 \\ \left(\Delta_{\alpha}+\sum_{\ell=1}^{n} \ell c_{\ell} \frac{\partial}{\partial c_{\ell}}\right)\left|I^{(n)}\right\rangle & \text { for } \quad k=0\end{cases}
$$

where $L_{k}$ are the generators of Virasoro algebra at central charge $c=1+6 Q^{2}$, and

$$
\Lambda_{k} \equiv\left\{\begin{array}{ll}
-\sum_{\ell=k-n}^{n} c_{\ell} c_{k-\ell} & \text { for } \quad n+1 \leq k \leq 2 n \\
-\sum_{\ell=1}^{n-1} c_{\ell} c_{n-\ell}+((k+1) Q-2 \alpha) c_{k} & \text { for } \quad 1 \leq k \leq n
\end{array} .\right.
$$

We call $\left|I^{(n)}\right\rangle$ satisfying the above equations an "irregular state" of rank $n$, or simply a rank- $n$ state. Note that the rank-zero state $\left|I^{(0)}\right\rangle$ is naturally identified with a regular primary state of Virasoro algebra.

Note here that $\left|I^{(n)}\right\rangle$ is a simultaneous eigenstate of $L_{n}, \cdots, L_{2 n-1}$ and $L_{2 n}$ with eigenvalues fixed by $c_{1}, \cdots, c_{n}$ and $\alpha .{ }^{15}$ This particularly implies that $L_{k}\left|I^{(n)}\right\rangle=0$ for all $k>2 n$. We particularly call $\alpha$ the "Liouville momentum" of $\left|I^{(n)}\right\rangle$, which is natural in the following sense. In [31], the rank- $n$ state $\left|I^{(n)}\right\rangle$ was constructed in a colliding limit of $n$ regular vertex operators $e^{2 \gamma \phi(z)}$ to a regular primary state $\left|\Delta_{\gamma_{0}}\right\rangle \cdot{ }^{16}$ Namely, $\left|I^{(n)}\right\rangle$ is obtained from

$$
e^{2 \gamma_{n} \phi\left(z_{n}\right)} e^{2 \gamma_{n-1} \phi\left(z_{n-1}\right)} \cdots e^{2 \gamma_{1} \phi\left(z_{1}\right)}\left|\Delta_{\gamma_{0}}\right\rangle,
$$

in the limit $z_{1}, \cdots, z_{n} \rightarrow 0$. For this limit to make sense, we need to take $\gamma_{0}, \cdots, \gamma_{n} \rightarrow \infty$ simultaneously with $\sum_{i=0}^{n} \gamma_{i}$ kept fixed. This sum of $\gamma_{i}$ is identified with $\alpha$ in (2.9), i.e., $\alpha \equiv \sum_{i=0}^{n} \gamma_{i}$. Therefore, $\alpha$ characterizing $\left|I^{(n)}\right\rangle$ is the sum of the Liouville momenta of regular vertex operators involved in the colliding limit. This point will be important in section 4.

On the other hand, $\left|I^{(n)}\right\rangle$ is not an eigenstate of $L_{0}, \cdots, L_{n-2}$ or $L_{n-1}$. Indeed, the actions of $L_{0}, \cdots, L_{n-1}$ involve derivatives with respect to $c_{i}$. This means that (2.9) are differential equations, and therefore $\left|I^{(n)}\right\rangle$ is not uniquely fixed by $c_{1}, \cdots, c_{n}$ and $\alpha$. To fix $\left|I^{(n)}\right\rangle$ completely, we need to specify its "boundary condition" or "asymptotic behavior." In this paper, we assume the following asymptotic behavior of $\left|I^{(n)}\right\rangle$ proposed in [31]; in the small $c_{n}$ limit, $\left|I^{(n)}\right\rangle$ behaves as

$$
\left|I^{(n)}(\alpha, \boldsymbol{c})\right\rangle=f\left(\alpha, \boldsymbol{c}, \beta_{n-1}\right)\left(\left|I^{(n-1)}\left(\beta_{n-1}, \widetilde{\boldsymbol{c}}\right)\right\rangle+\sum_{k=1}^{\infty}\left(c_{n}\right)^{k}\left|I_{k}^{(n-1)}\right\rangle\right),
$$

\footnotetext{
${ }^{15}$ To be precise, eigenstates of $L_{n}, \cdots, L_{2 n}$ were considered in [29, 31], while [30] studied eigenstates of $L_{1}$ and $L_{2 n}$. The relation between these two were discussed in appendix A of [32]. These two descriptions are both useful in understanding these special states.

${ }^{16}$ See section 2.2 in particular. See also section 2 of [56] for a review.
} 
where $\boldsymbol{c} \equiv\left(c_{1}, \cdots, c_{n}\right), \widetilde{\boldsymbol{c}} \equiv\left(c_{1}, \cdots, c_{n-1}\right)$, and $\beta_{n-1}$ is a free parameter characterizing the asymptotic behavior. ${ }^{17}$ On the r.h.s. of $(2.12)$, the leading term $\left|I^{(n-1)}\left(\beta_{n-1}, \widetilde{\boldsymbol{c}}\right)\right\rangle$ is the rank- $(n-1)$ irregular state satisfying (2.9) with $n$ and $\alpha$ replaced by $(n-1)$ and $\beta_{n-1}$, respectively. Note that $\beta_{n-1}$ is the Liouville momentum of this leading term. On the other hand, the remaining terms $\left|I_{k}^{(n-1)}\right\rangle$ are generalized descendants of $\left|I^{(n-1)}\left(\beta_{n-1}, \widetilde{c}\right)\right\rangle$. Here, generalized descendants are descendants in the sense of Virasoro generators $L_{k<0}$ and the derivatives with respect to $c_{1}, \cdots, c_{n-1} \cdot{ }^{18}$ The prefactor $f\left(\alpha, \boldsymbol{c}, \beta_{n-1}\right)$ in $(2.12)$ is a function necessary for the ansatz (2.12) to satisfy the differential equations (2.9) order by order in $c_{n}{ }^{19}$

Note here that $\left|I^{(n-1)}\left(\beta_{n-1}, \widetilde{\boldsymbol{c}}\right)\right\rangle$ on the r.h.s. of (2.12) can further be expanded in terms of rank- $(n-2)$ state and its generalized descendants. In this expansion, the Liouville momentum of the rank- $(n-2)$ state is again a free parameter, which we denote by $\beta_{n-2}$. Continuing this expansion until we reach the rank-zero state (i.e., regular primary state), we see that there are $n$ free parameters $\beta_{0}, \cdots, \beta_{n-1}$ characterizing the original rank- $n$ state $\left|I^{(n)}\right\rangle$. This means that the rank- $n$ state $\left|I^{(n)}\right\rangle$ depends not only on $\alpha$ and $\boldsymbol{c}=\left(c_{1}, \cdots, c_{n}\right)$ but also on $\beta \equiv\left(\beta_{0}, \cdots, \beta_{n-1}\right)$. In particular, $\beta=\left(\beta_{0}, \cdots, \beta_{n-1}\right)$ are parameters completely fixing the asymptotic behavior of $\left|I^{(n)}\right\rangle$. Therefore, in the rest of this paper, we write $\left|I^{(n)}(\alpha, \boldsymbol{c} ; \boldsymbol{\beta})\right\rangle$ instead of $\left|I^{(n)}(\alpha, \boldsymbol{c})\right\rangle$ to denote the Liouville irregular state of rank $n$. This asymptotic expansion of the rank- $n$ irregular state is proposed in [31] based on the colliding limit of regular vertex operators explained around (2.11).

Given the above correspondence between irregular punctures and Liouville irregular states, the partition functions of the $\left(A_{1}, A_{2 n-3}\right)$ and $\left(A_{1}, D_{2 n}\right)$ theories are expected to be identified with Liouville correlators involving an irregular state. In particular, since $\mathcal{C}$ for the $\left(A_{1}, A_{2 n-3}\right)$ theory is a sphere with one irregular puncture of rank $n$, its partition function is expected to be written as

$$
\mathcal{Z}_{\left(A_{1}, A_{2 n-3}\right)}^{\epsilon_{1}, \epsilon_{2}}=\left\langle 0 \mid I^{(n)}(\alpha, \boldsymbol{c} ; \boldsymbol{\beta})\right\rangle,
$$

where $|0\rangle$ is the vacuum. On the other hand, $\mathcal{C}$ for the $\left(A_{1}, D_{2 n}\right)$ theory is a sphere with one regular puncture and one irregular puncture of rank $n$, and therefore its partition function is expected to be given by

$$
\mathcal{Z}_{\left(A_{1}, D_{2 n}\right)}^{\epsilon_{1}, \epsilon_{2}}=\left\langle\Delta_{\gamma} \mid I^{(n)}(\alpha, \boldsymbol{c} ; \boldsymbol{\beta})\right\rangle .
$$

In the next section, we explicitly identify the irregular states of rank 2 and 3 , and then evaluate the partition functions for $\left(A_{1}, A_{3}\right)$ and $\left(A_{1}, D_{4}\right)$ theories via the relations $(2.13)$ and (2.14).

\section{Partition functions from irregular states}

In this section, we explicitly compute the Liouville irregular state of rank 2 and 3 , and then evaluate the partition functions of the $\left(A_{1}, A_{3}\right)$ and $\left(A_{1}, D_{4}\right)$ theories via (2.13) and (2.14).

\footnotetext{
${ }^{17}$ See in particular section 3.2 and appendix B.2 of the reference [31] for the first discussion of this ansatz. We also mention here that $\left|I_{k}^{(n-1)}\right\rangle$ in (2.12) was denoted as $\left|I_{n k}^{(n-1)}\right\rangle$ in [31]. We use $\left|I_{k}^{(n)}\right\rangle$ instead of $\left|I_{n k}^{(n)}\right\rangle$ to reduce clutter.

${ }^{18}$ These derivatives make sense since $\left|I^{(n-1)}\left(\beta_{n-1}, \widetilde{\boldsymbol{c}}\right)\right\rangle$ depends on $c_{1}, \cdots, c_{n-1}$.

${ }^{19}$ For concrete expressions for $f(\alpha, \boldsymbol{c} ; \beta)$ for $n=2$ and 3 , see (3.9) and (3.40) in section 3 .
} 


\section{1 $\left(A_{1}, D_{4}\right)$ theory from rank-2 irregular state}

Let us first focus on the rank-2 irregular state and the $\left(A_{1}, D_{4}\right)$ theory. As reviewed in the previous section, the partition function of the $\left(A_{1}, D_{4}\right)$ theory is expected to be given by

$$
Z_{\left(A_{1}, D_{4}\right)}^{\epsilon_{1}, \epsilon_{2}}=\left\langle\Delta_{\gamma} \mid I^{(2)}(\alpha, \boldsymbol{c} ; \boldsymbol{\beta})\right\rangle,
$$

where $\left|\Delta_{\gamma}\right\rangle$ is a regular primary state of holomorphic dimension $\Delta_{\gamma} \equiv \gamma(Q-\gamma), \boldsymbol{c}$ and $\boldsymbol{\beta}$ are respectively short-hand notations for $\left(c_{1}, c_{2}\right)$ and $\left(\beta_{0}, \beta_{1}\right)$, and $\left|I^{(2)}(\alpha, \boldsymbol{c} ; \boldsymbol{\beta})\right\rangle$ is the irregular state of rank two satisfying

$$
\begin{aligned}
L_{4}\left|I^{(2)}(\alpha, \boldsymbol{c} ; \boldsymbol{\beta})\right\rangle & =-c_{2}^{2}\left|I^{(2)}(\alpha, \boldsymbol{c} ; \boldsymbol{\beta})\right\rangle, \\
L_{3}\left|I^{(2)}(\alpha, \boldsymbol{c} ; \boldsymbol{\beta})\right\rangle & =-2 c_{1} c_{2}\left|I^{(2)}(\alpha, \boldsymbol{c} ; \boldsymbol{\beta})\right\rangle, \\
L_{2}\left|I^{(2)}(\alpha, \boldsymbol{c} ; \boldsymbol{\beta})\right\rangle & =-\left(c_{1}^{2}+c_{2}(2 \alpha-3 Q)\right)\left|I^{(2)}(\alpha, \boldsymbol{c} ; \boldsymbol{\beta})\right\rangle, \\
L_{1}\left|I^{(2)}(\alpha, \boldsymbol{c} ; \boldsymbol{\beta})\right\rangle & =\left(c_{2} \frac{\partial}{\partial c_{1}}-2 c_{1}(\alpha-Q)\right)\left|I^{(2)}(\alpha, \boldsymbol{c} ; \boldsymbol{\beta})\right\rangle, \\
L_{0}\left|I^{(2)}(\alpha, \boldsymbol{c} ; \boldsymbol{\beta})\right\rangle & =\left(\Delta_{\alpha}+c_{1} \frac{\partial}{\partial c_{1}}+2 c_{2} \frac{\partial}{\partial c_{2}}\right)\left|I^{(2)}(\alpha, \boldsymbol{c} ; \boldsymbol{\beta})\right\rangle .
\end{aligned}
$$

To identify the relation between the $4 \mathrm{~d}$ and $2 \mathrm{~d}$ parameters, let us first compute the $\mathrm{SW}$ curve in terms of the irregular state using the identification (2.8). Since the Virasoro stress tensor is expanded as $T(z)=\sum_{n=-\infty}^{\infty} L_{n} z^{-n-2}$, the above equations and $\left\langle\Delta_{\gamma}\right| L_{0}=\left\langle\Delta_{\gamma}\right| \Delta_{\gamma}$ imply that

$$
\frac{\left\langle\Delta_{\gamma}|T(z)| I^{(2)}(\alpha, \boldsymbol{c} ; \boldsymbol{\beta})\right\rangle}{\left\langle\Delta_{\gamma} \mid I^{(2)}(\alpha, \boldsymbol{c} ; \boldsymbol{\beta})\right\rangle}=\frac{\Delta_{\gamma}}{z^{2}}+\frac{\sqrt{-i c_{2}} u}{z^{3}}-\frac{c_{1}^{2}+c_{2}(2 \alpha-3 Q)}{z^{4}}-\frac{2 c_{1} c_{2}}{z^{5}}-\frac{c_{2}^{2}}{z^{6}},
$$

where $u \equiv-\sqrt{-i c_{2}} \frac{\partial}{\partial c_{1}} \log \left\langle\Delta_{\gamma} \mid I^{(2)}(\alpha, \beta, c)\right\rangle-\frac{2 c_{1}}{\sqrt{i c_{2}}}(\alpha-Q)$. To make a connection to the SW curve (2.5) of the $\left(A_{1}, D_{4}\right)$ theory, let us rescale the coordinate as $z \rightarrow \sqrt{-i c_{2}} z$. Since the stress tensor $T$ is of dimension two, this also rescales $T$ as $T \rightarrow-\left(c_{2}\right)^{-2} T{ }^{20}$ As a result, we have

$$
\frac{\left\langle\Delta_{\gamma}|T(z)| I^{(2)}(\alpha, \boldsymbol{c} ; \boldsymbol{\beta})\right\rangle}{\left\langle\Delta_{\gamma} \mid I^{(2)}(\alpha, \boldsymbol{c} ; \boldsymbol{\beta})\right\rangle}=\frac{\Delta_{\gamma}}{z^{2}}+\frac{u}{z^{3}}+\frac{\frac{c_{1}^{2}}{c_{2}}+(2 \alpha-3 Q)}{z^{4}}+\sqrt{\frac{i c_{1}^{2}}{c_{2}}} \frac{1}{z^{5}}+\frac{1}{z^{6}} .
$$

The identification (2.8) then gives us exactly the same curve as the SW-curve (2.5) of the $\left(A_{1}, D_{4}\right)$ theory. From this curve, we can read off how the $2 \mathrm{~d}$ parameters are related to the four-dimensional mass parameters, relevant coupling, and the vev of the Coulomb branch operator. In particular, we see that $\sqrt{i c_{1}^{2} / c_{2}}$ is identified with the relevant coupling of dimension $1 / 2$.

\subsubsection{Ansatz in terms of generalized descendants}

Given the identification of parameters, we now compute the rank-2 irregular state $\left|I^{(2)}(\alpha, \boldsymbol{c} ; \boldsymbol{\beta})\right\rangle$. To that end, we use the ansatz (2.12) for the irregular state reviewed in

\footnotetext{
${ }^{20}$ In other words, $T(z) d z$ is invariant under this transformation.
} 
section 2. For the rank-2 irregular state, the ansatz is written as

$$
\left|I^{(2)}(\alpha, \boldsymbol{c} ; \boldsymbol{\beta})\right\rangle=c_{1}^{\nu_{1}} c_{2}^{\nu_{2}} e^{\left(\alpha-\beta_{1}\right) \frac{c_{1}^{2}}{c_{2}}}\left(\left|I^{(1)}\left(\beta_{1}, c_{1} ; \beta_{0}\right)\right\rangle+\sum_{k=1}^{\infty}\left(c_{2}\right)^{k}\left|I_{k}^{(1)}\right\rangle\right),
$$

where the prefactor $f\left(\alpha, \boldsymbol{c}, \beta_{1}\right)=c_{1}^{\nu_{1}} c_{2}^{\nu_{2}} e^{\left(\alpha-\beta_{1}\right) \frac{c_{1}^{2}}{c_{2}}}$ was identified in [31]. The states $\left|I_{k}^{(1)}\right\rangle$ are generalized descendants of the rank-one irregular state $\left|I^{(1)}\left(\beta_{1}, c_{1} ; \beta_{0}\right)\right\rangle .{ }^{21}$ As reviewed in section 2 , a generalized descendant is a descendant of $\left|I^{(1)}\left(\beta_{1}, c_{1} ; \beta_{0}\right)\right\rangle$ in the sense of $L_{-\ell}$ and $\partial / \partial c_{1}$, i.e., a linear combination of states of the form

$$
\left(L_{-\ell_{1}}\right)^{p_{1}}\left(L_{-\ell_{2}}\right)^{p_{2}} \cdots\left(L_{-\ell_{n}}\right)^{p_{n}} \frac{\partial^{m}}{\partial c_{1}^{m}}\left|I^{(1)}\left(\beta_{1}, c_{1} ; \beta_{0}\right)\right\rangle,
$$

for integers $m, n \geq 0, p_{i}>0$, and $\ell_{1}>\ell_{2}>\cdots>\ell_{n}>0$. The coefficients of this linear combination turn out to depend on $c_{1}$ and $\beta_{1}$ but are independent of $\beta_{0}$. As pointed out in [31], $\left|I_{k}^{(1)}\right\rangle$ turns out to be a level- $2 k$ generalized descendant, where $L_{-k}, \partial^{k} / \partial c_{1}^{k}$ and $1 / c_{1}^{k}$ are regarded as level $k$. The exponents $\nu_{1}$ and $\nu_{2}$ in (3.9) are fixed as [31]

$$
\nu_{1}=2\left(\alpha-\beta_{1}\right)\left(Q-\beta_{1}\right), \quad \nu_{2}=\frac{1}{2}\left(\beta_{1}-\alpha\right)\left(3 Q-3 \beta_{1}-\alpha\right),
$$

in order for the ansatz (3.9) to satisfy (3.2)-(3.6). Note here that the ansatz (3.9) particularly implies that in the limit $c_{2} \rightarrow 0$ the irregular state behaves as $\left|I^{(2)}(\alpha, \boldsymbol{c} ; \boldsymbol{\beta})\right\rangle \rightarrow$ $c_{1}^{\nu_{1}} c_{2}^{\nu_{2}} e^{\left(\alpha-\beta_{1}\right) \frac{c_{1}^{2}}{c_{2}}}\left|I^{(1)}\left(\beta_{1}, c_{1} ; \beta_{0}\right)\right\rangle$. Therefore (3.9) fixes the "boundary condition" for $\left|I^{(2)}(\alpha, \boldsymbol{c} ; \boldsymbol{\beta})\right\rangle$ at $c_{2}=0$. The authors of [31] conjectured that (3.2)-(3.6) uniquely determine the generalized descendants $\left|I_{k}^{(1)}\left(\alpha, c_{1} ; \boldsymbol{\beta}\right)\right\rangle$.

We now explicitly compute $\left|I_{k}^{(1)}\left(\alpha, c_{1} ; \beta\right)\right\rangle$ by solving (3.2)-(3.6) order by order of $c_{2}$. Since the basic strategy is already discussed in [31], we move the detailed description for the order-by-order computation to appendix A.1, and write down the results here:

$$
\begin{aligned}
\left|I_{0}^{(1)}\right\rangle & =\left|I^{(1)}\left(\beta_{1}, c_{1} ; \beta_{0}\right)\right\rangle \\
\left|I_{1}^{(1)}\right\rangle & =\left(\frac{1}{2 c_{1}} L_{-1}+\frac{2 \alpha-3 \beta_{1}}{2 c_{1}} \partial_{c_{1}}+\frac{\nu_{3}}{c_{1}^{2}}\right)\left|I^{(1)}\left(\beta_{1}, c_{1} ; \beta_{0}\right)\right\rangle \\
\left|I_{2}^{(1)}\right\rangle & =\left(-\frac{1}{4 c_{1}^{2}} L_{-2}+\frac{1}{8 c_{1}^{2}} L_{-1}^{2}+\frac{2 \alpha-3 \beta_{1}}{4 c_{1}^{2}} L_{-1} \partial_{c_{1}}\right. \\
& +\frac{2\left(2 \alpha-3 \beta_{1}\right)^{2}-1}{16 c_{1}^{2}} \partial_{c_{1}}^{2}+\frac{11 \beta_{1}-8 \alpha+Q+4 \nu_{3}}{8 c_{1}^{3}} L_{-1} \\
& \left.+\frac{-4 \alpha^{2}+8 \alpha\left(3 \beta_{1}+\nu_{3}\right)-\beta_{1}\left(23 \beta_{1}+12 \nu_{3}\right)+Q\left(-12 \alpha+11 \beta_{1}\right)}{8 c_{1}^{3}} \partial_{c_{1}}+\frac{\nu_{4}}{c_{1}^{4}}\right)\left|I^{(1)}\left(\beta_{1}, c_{1} ; \beta_{0}\right)\right\rangle
\end{aligned}
$$

where $\partial_{c_{1}} \equiv \partial / \partial c_{1}$, and $\nu_{3}$ and $\nu_{4}$ are given by

$$
\begin{aligned}
\nu_{3} \equiv & \frac{1}{2}\left(3 Q+\alpha-3 \beta_{1}\right)\left(\alpha-\beta_{1}\right)\left(Q-\beta_{1}\right), \\
\nu_{4} \equiv & \frac{\left(Q-\beta_{1}\right)\left(\alpha-\beta_{1}\right)}{8}\left(\left(\alpha-\beta_{1}\right)^{3}\left(Q-\beta_{1}\right)+\left(\alpha-\beta_{1}\right)^{2}\left(4 \beta_{1}^{2}+6 Q^{2}-10 \beta_{1} Q-2\right)\right. \\
& \left.+\left(\alpha-\beta_{1}\right)\left(3 Q-2 \beta_{1}\right)\left(2 \beta_{1}^{2}+3 Q^{2}-5 \beta_{1} Q-5\right)-8 \beta_{1}^{2}-21 Q^{2}+26 \beta_{1} Q+1\right) .
\end{aligned}
$$

\footnotetext{
${ }^{21}$ As mentioned already, we here use a slightly different convention for $\left|I_{k}^{(2)}\right\rangle$. Namely, $\left|I_{k}^{(2)}\right\rangle$ here is identified with $\left|I_{2 k}^{(2)}\right\rangle$ in [31].
} 
From the fact that $\left|I^{(1)}\left(\beta_{1}, c_{1} ; \beta_{0}\right)\right\rangle$ is an irregular state of rank one, we see that (3.9) with the above expressions substituted correctly satisfies the equations (3.2)-(3.6).

\subsubsection{Irregular conformal block}

Using the above result for the irregular state of rank two, we here evaluate the $c_{2}$-expansion of the inner product $\left\langle\Delta_{\gamma} \mid I^{(2)}(\alpha, \boldsymbol{c} ; \beta)\right\rangle$. We particularly show that, in the limit of $Q \rightarrow 0$, this $c_{2}$-expansion is precisely identical to the strong coupling expansion of the partition function of the $\left(A_{1}, D_{4}\right)$ theory obtained in [15].

To that end, we first evaluate the inner product of (3.9) and $\left|\Delta_{\gamma}\right\rangle$, which reduces to computing the inner product $\left\langle\Delta_{\gamma} \mid I_{k}^{(1)}\right\rangle$ for all $k$. Note here that, since $\left\langle\Delta_{\gamma}\right| L_{-n}=0$ for $n>1$, we only need to keep track of terms without $L_{-n}$ in (3.12)-(3.14). Moreover, since the rank-one irregular state satisfies $L_{0}\left|I^{(1)}\left(\beta_{1}, c_{1} ; \beta_{0}\right)\right\rangle=\left(\Delta_{\beta_{1}}+c_{1} \partial_{c_{1}}\right)\left|I^{(1)}\left(\beta_{1}, c_{1} ; \beta_{0}\right)\right\rangle$, the action of $\partial_{c_{1}}$ on $\left|I^{(1)}\left(\beta_{1}, c_{1} ; \beta_{0}\right)\right\rangle$ is translated into the action of $L_{0}$. Therefore,

$$
\left\langle\Delta_{\gamma}\left|\left(c_{1} \partial_{c_{1}}\right)^{k}\right| I^{(1)}\left(\beta_{1}, c_{1} ; \beta_{0}\right)\right\rangle=\left(\Delta_{\gamma}-\Delta_{\beta_{1}}\right)^{k}\left\langle\Delta_{\gamma} \mid I^{(1)}\left(\beta_{1}, c_{1} ; \beta_{0}\right)\right\rangle .
$$

Using this identity, we see that the inner product of $\left|I^{(2)}(\alpha, \beta, c)\right\rangle$ and $\left|\Delta_{\gamma}\right\rangle$ is written as

$$
\left\langle\Delta_{\gamma} \mid I^{(2)}(\alpha, \boldsymbol{c} ; \beta)\right\rangle=\left\langle\Delta_{\gamma} \mid I^{(1)}\left(\beta_{1}, c_{1} ; \beta_{0}\right)\right\rangle c_{1}^{\nu_{1}} c_{2}^{\nu_{2}} e^{\left(\alpha-\beta_{1}\right) \frac{c_{1}^{2}}{c_{2}}} \sum_{k=0}^{\infty}\left(\frac{c_{2}}{i c_{1}^{2}}\right)^{k} D_{k}\left(\alpha, \beta_{1}, \gamma\right),
$$

with coefficients $D_{k}$ depending on $\alpha, \beta_{1}$ and $\gamma$. We here show the expressions for the first several coefficients:

$$
\begin{aligned}
& D_{0}=1 \\
& D_{1}=\frac{i}{2}\left(\left(2 \alpha-3 \beta_{1}\right) \Delta_{\gamma}+\left(Q-\beta_{1}\right)\left(6 \beta_{1}^{2}-3 \beta_{1}(2 \alpha+Q)+\alpha(\alpha+3 Q)\right)\right) \\
& D_{2}=\frac{1}{16}\left\{\Delta _ { \gamma } \left(\left(1-2\left(2 \alpha-3 \beta_{1}\right)^{2}\right) \Delta_{\gamma}-72 \beta_{1}^{4}-6 \beta_{1}^{2}\left(10 \alpha^{2}+6 Q^{2}+30 \alpha Q-11\right)\right.\right. \\
& \quad+4 \beta_{1}\left(2 \alpha^{3}+3 \alpha\left(5 Q^{2}-6\right)+21 \alpha^{2} Q-6 Q\right)-8 \alpha\left(Q\left(\alpha^{2}+3 \alpha Q-3\right)-2 \alpha\right) \\
& \left.\quad+12 \beta_{1}^{3}(10 \alpha+9 Q)-1\right)-\left(Q-\beta_{1}\right)\left(3 \beta _ { 1 } \left(-24 \beta_{1}^{4}+5 \beta_{1}^{2}\left(7-6 Q^{2}\right)+\beta_{1} Q\left(6 Q^{2}-35\right)\right.\right. \\
& \left.\quad+14 Q^{2}+48 \beta_{1}^{3} Q-1\right)+6 \alpha^{2}\left(-16 \beta_{1}^{3}+8 \beta_{1}+3 Q^{3}-17 \beta_{1} Q^{2}+5\left(6 \beta_{1}^{2}-1\right) Q\right) \\
& \quad-2 \alpha\left(-72 \beta_{1}^{4}+70 \beta_{1}^{2}+18 \beta_{1} Q^{3}+\left(21-90 \beta_{1}^{2}\right) Q^{2}+4\left(36 \beta_{1}^{2}-17\right) \beta_{1} Q-1\right) \\
& \left.\left.\quad+2 \alpha^{4}\left(Q-\beta_{1}\right)+4 \alpha^{3}\left(3\left(Q-2 \beta_{1}\right)\left(Q-\beta_{1}\right)-1\right)\right)\right\}
\end{aligned}
$$

Some more coefficients for higher orders are shown in appendix A.1.

Let us now consider the prefactor $\left\langle\Delta_{\gamma} \mid I^{(1)}\left(\beta_{1}, c_{1} ; \beta_{0}\right)\right\rangle$ in (3.18). Here $\left|I^{(1)}\left(\beta_{1}, c_{1} ; \beta_{0}\right)\right\rangle$ is a rank-one irregular state satisfying (2.9) for $n=1$. In the case of $\beta_{0}=\beta_{1}$, the $c_{1}$-expansion of $\left|I^{(1)}\left(\beta_{1}, c_{1} ; \beta_{0}\right)\right\rangle$ was studied in [29], whose generalization to $\beta_{0} \neq \beta_{1}$ is of the form

$$
\left|I^{(1)}\left(\beta_{1}, c_{1} ; \beta_{0}\right)\right\rangle=c_{1}^{\Delta_{\beta_{1}}-\Delta_{\beta_{0}}}\left(\left|\Delta_{\beta_{0}}\right\rangle+\sum_{k=1}^{\infty}\left(c_{1}\right)^{k}\left|R_{k}\right\rangle\right),
$$

where the $\left|R_{k}\right\rangle$ is a level- $k$ Virasoro descendant of $\left|\Delta_{\beta_{0}}\right\rangle \cdot{ }^{22}$ While the Virasoro descendants $\left|R_{k}\right\rangle$ are fixed by $\beta_{0}$ and $\beta_{1}$ so that $\left|I^{(1)}\left(\beta_{1}, c_{1} ; \beta_{0}\right)\right\rangle$ satisfies $(2.9)$, we do not need to

\footnotetext{
${ }^{22}$ Note here that the expression (3.22) fixes the "boundary condition" for $\left|I^{(1)}\left(\beta_{1}, c_{1} ; \beta_{0}\right)\right\rangle$ at $c_{1}=0$.
} 
evaluate them explicitly. Indeed, since $\left|R_{k}\right\rangle$ are all Virasoro descendants, we see that

$$
\left\langle\Delta_{\gamma} \mid I^{(1)}\left(\beta_{1}, c_{1} ; \beta_{0}\right)\right\rangle=\left\{\begin{array}{ll}
c_{1}^{\Delta_{\beta_{1}}-\Delta_{\beta_{0}}} & \left(\Delta_{\beta_{0}}=\Delta_{\gamma}\right) \\
0 & \left(\Delta_{\beta_{0}} \neq \Delta_{\gamma}\right)
\end{array} .\right.
$$

For this inner product to be non-vanishing, we set $\gamma=\beta_{0}$ in the rest of this sub-section.

Substituting (3.23) in (3.18), we obtain an explicit expression for $\left\langle\Delta_{\beta_{0}} \mid I^{(2)}(\alpha, \boldsymbol{c} ; \boldsymbol{\beta})\right\rangle$. This is then identified via (2.14) with the partition function of the $\left(A_{1}, D_{4}\right)$ theory, i.e.,

$$
\mathcal{Z}_{\left(A_{1}, D_{4}\right)}^{\epsilon_{1}, \epsilon_{2}}=c_{1}^{2\left(\alpha-\beta_{1}\right)\left(Q-\beta_{1}\right)+\Delta_{\beta_{0}}-\Delta_{\alpha}} c_{2}^{\frac{1}{2}\left(\beta_{1}-\alpha\right)\left(3 Q-3 \beta_{1}-\alpha\right)} e^{\left(\alpha-\beta_{1}\right) \frac{c_{1}^{2}}{c_{2}}} \sum_{k=0}^{\infty}\left(\frac{c_{2}}{i c_{1}^{2}}\right)^{k} D_{k}\left(\alpha, \beta_{1}, \beta_{0}\right),
$$

up to a $c_{i}$-independent prefactor, where the coefficients $D_{i}$ are those in (3.19)-(3.21) with $\gamma=\beta_{0}$. Recall here that $\sqrt{i c_{1}^{2} / c_{2}}$ is identified with the relevant coupling of dimension $1 / 2$ of the $\left(A_{1}, D_{4}\right)$ theory. Therefore, (3.24) is an expansion of the $\left(A_{1}, D_{4}\right)$ partition function in the inverse powers of the relevant coupling, i.e., the strong coupling expansion of $\mathcal{Z}_{\left(A_{1}, D_{4}\right.}^{\epsilon_{1}, \epsilon_{2}}$.

Let us now compare (3.24) with the $\left(A_{1}, D_{4}\right)$ partition function obtained in [15]. ${ }^{23}$ Since the expression obtained in [15] is for the case $Q=0$, we will focus on the $Q \rightarrow 0$ limit of (3.24) in the rest of this sub-section. Note that $Q=0$ corresponds to $\epsilon_{1}+\epsilon_{2}=0$ in four dimensions. We first change the variables from $\left(c_{2} / i c_{1}^{2}, \alpha, \beta_{0}, \beta_{1}\right)$ to $\left(s, \nu, \theta_{s}, \theta_{t}\right)$ by

$$
\frac{c_{2}}{i c_{1}^{2}} \equiv \frac{1}{s}, \quad \alpha \equiv i\left(\theta_{s}+\theta_{t}\right), \quad \beta_{0} \equiv i\left(\theta_{t}-\theta_{s}\right), \quad \beta_{1} \equiv \frac{i\left(\theta_{s}+\theta_{t}-3 \nu\right)}{3} .
$$

Note that these relations could receive $\mathcal{O}(Q)$ corrections, as we will discuss in section 4 . We omit such $\mathcal{O}(Q)$ corrections for the moment since we here focus on the limit $Q \rightarrow 0$. With the above change of variables, the partition function for $Q=0$ is written as

$$
\left.\mathcal{Z}_{\left(A_{1}, D_{4}\right)}^{\epsilon_{1}, \epsilon_{2}}\right|_{\epsilon_{1}+\epsilon_{2}=0}=c_{1}^{-\frac{\left(3 \nu+2\left(\theta_{s}+\theta_{t}\right)\right)^{2}}{9}} c_{2}^{-\frac{3 \nu^{2}}{2}+\frac{2\left(\theta_{s}+\theta_{t}\right)^{2}}{3}} e^{\nu s+\frac{2}{3}\left(\theta_{s}+\theta_{t}\right) s} \sum_{k=0}^{\infty} \frac{\left.D_{k}\right|_{Q=0}}{s^{k}} .
$$

We see that this expression is identical to $\mathcal{G}(\nu, s)$ in eq. (3.48) of [15] obtained via the connection to Painlevé IV, up to an $s$-independent prefactor. In particular, the asymptotic behavior, $e^{\nu s+\frac{2}{3}\left(\theta_{s}+\theta_{t}\right) s}$, in the limit $s \rightarrow \infty$ is precisely identical. Moreover, our $\left.D_{1}\right|_{Q=0}$ and $\left.D_{2}\right|_{Q=0}$ are written as

$$
\begin{aligned}
\left.D_{1}\right|_{Q=0}= & 3 \nu^{3}-2 \nu\left(\theta_{s}^{2}-\theta_{s} \theta_{t}+\theta_{t}^{2}\right)-\frac{2}{9}\left(2 \theta_{s}-\theta_{t}\right)\left(\theta_{s}+\theta_{t}\right)\left(\theta_{s}-2 \theta_{t}\right) \\
\left.D_{2}\right|_{Q=0}= & \frac{9 \nu^{6}}{2}+\nu^{4}\left(\frac{105}{16}-6\left(\theta_{s}^{2}-\theta_{s} \theta_{t}+\theta_{t}^{2}\right)\right)-\frac{2}{3} \nu^{3}\left(\theta_{s}-2 \theta_{t}\right)\left(2 \theta_{s}-\theta_{t}\right)\left(\theta_{s}+\theta_{t}\right) \\
& +\nu^{2}\left(\frac{1}{2}\left(\theta_{s}^{2}-\theta_{s} \theta_{t}+\theta_{t}^{2}\right)\left(4\left(\theta_{s}^{2}-\theta_{s} \theta_{t}+\theta_{t}^{2}\right)-11\right)+\frac{3}{16}\right) \\
& +\frac{\nu}{9}\left(2 \theta_{s}-\theta_{t}\right)\left(\theta_{s}+\theta_{t}\right)\left(\theta_{s}-2 \theta_{t}\right)\left(4\left(\theta_{s}^{2}-\theta_{s} \theta_{t}+\theta_{t}^{2}\right)-7\right) \\
& +\frac{1}{324}\left(32 \theta_{s}^{6}-96 \theta_{s}^{5} \theta_{t}+\theta_{s}^{4}\left(36-24 \theta_{t}^{2}\right)+8 \theta_{s}^{3} \theta_{t}\left(26 \theta_{t}^{2}-9\right)-3 \theta_{s}^{2}\left(8 \theta_{t}^{4}-36 \theta_{t}^{2}+9\right)\right. \\
& \left.\quad-3 \theta_{s} \theta_{t}\left(32 \theta_{t}^{4}+24 \theta_{t}^{2}-9\right)+\theta_{t}^{2}\left(32 \theta_{t}^{4}+36 \theta_{t}^{2}-27\right)\right),
\end{aligned}
$$

\footnotetext{
${ }^{23}$ In this reference, the $\left(A_{1}, D_{4}\right)$ theory is called the $H_{2}$ Argyres-Douglas theory.
} 
which are precisely identical to $D_{1}$ and $D_{2}$ in eq. (3.49) of [15]. Therefore, the $Q \rightarrow 0$ limit of our partition function $\mathcal{Z}_{\left(A_{1}, D_{4}\right)}^{\epsilon_{1}, \epsilon_{2}}$ is identical to $\mathcal{G}(\nu, s)$ in [15] up to a prefactor. In particular, the strong coupling expansion in powers of $1 / s$ corresponds to the $c_{2}$-expansion of the irregular state $\left|I^{(2)}(\alpha, \boldsymbol{c} ; \boldsymbol{\beta})\right\rangle$ arising from the ansatz (3.9).

Note here that the above $Q \rightarrow 0$ limits of $D_{1}$ and $D_{2}$ were also obtained in [24], with a different parameterization of the irregular conformal block. In particular, the expansion parameter $1 / s$ is identified in [24] as the coordinate of the vertex operator corresponding to the regular singularity. On the other hand, we here identify the expansion in powers of $1 / \mathrm{s}$ as the asymptotic $c_{2}$-expansion arising from the ansatz (3.9) proposed in [31]. These two parameter identifications are expected to be related by a change of coordinate on sphere. We have also evaluated the $Q$-dependent terms as shown in (3.21), which will be important in our discussion in section 4 .

\section{$3.2\left(A_{1}, A_{3}\right)$ theory from rank-3 irregular state}

Let us now turn to the $\left(A_{1}, A_{3}\right)$ theory. According to the generalized AGT correspondence, the partition function of the $\left(A_{1}, A_{3}\right)$ theory is expected to be given by

$$
Z_{\left(A_{1}, A_{3}\right)}^{\epsilon_{1}, \epsilon_{2}}=\left\langle 0 \mid I^{(3)}(\alpha, \boldsymbol{c} ; \boldsymbol{\beta})\right\rangle,
$$

where $|0\rangle$ is the vacuum state, and $\left|I^{(3)}(\alpha, \boldsymbol{c} ; \beta)\right\rangle$ is an irregular primary state of rankthree. Here $\boldsymbol{c} \equiv\left(c_{1}, c_{2}, c_{3}\right)$ and $\beta \equiv\left(\beta_{0}, \beta_{1}, \beta_{2}\right)$. This irregular state satisfies the following equations:

$$
\begin{aligned}
L_{6}\left|I^{(3)}(\alpha, \boldsymbol{c} ; \boldsymbol{\beta})\right\rangle & =-c_{3}^{2}\left|I^{(3)}(\alpha, \boldsymbol{c} ; \boldsymbol{\beta})\right\rangle \\
L_{5}\left|I^{(3)}(\alpha, \boldsymbol{c} ; \boldsymbol{\beta})\right\rangle & =-2 c_{2} c_{3}\left|I^{(3)}(\alpha, \boldsymbol{c} ; \boldsymbol{\beta})\right\rangle \\
L_{4}\left|I^{(3)}(\alpha, \boldsymbol{c} ; \boldsymbol{\beta})\right\rangle & =-\left(c_{2}^{2}+2 c_{3} c_{1}\right)\left|I^{(3)}(\alpha, \boldsymbol{c} ; \boldsymbol{\beta})\right\rangle \\
L_{3}\left|I^{(3)}(\alpha, \boldsymbol{c} ; \boldsymbol{\beta})\right\rangle & =-2\left(c_{1} c_{2}+c_{3}(\alpha-2 Q)\right)\left|I^{(3)}(\alpha, \boldsymbol{c} ; \boldsymbol{\beta})\right\rangle, \\
L_{2}\left|I^{(3)}(\alpha, \boldsymbol{c} ; \boldsymbol{\beta})\right\rangle & =\left(c_{3} \frac{\partial}{\partial c_{1}}-c_{2}(2 \alpha-3 Q)-c_{1}^{2}\right)\left|I^{(3)}(\alpha, \boldsymbol{c} ; \boldsymbol{\beta})\right\rangle, \\
L_{1}\left|I^{(3)}(\alpha, \boldsymbol{c} ; \boldsymbol{\beta})\right\rangle & =\left(2 c_{3} \frac{\partial}{\partial c_{2}}+c_{2} \frac{\partial}{\partial c_{1}}-2 c_{1}(\alpha-Q)\right)\left|I^{(3)}(\alpha, \boldsymbol{c} ; \boldsymbol{\beta})\right\rangle, \\
L_{0}\left|I^{(3)}(\alpha, \boldsymbol{c} ; \boldsymbol{\beta})\right\rangle & =\left(\Delta_{\alpha}+c_{1} \frac{\partial}{\partial c_{1}}+2 c_{2} \frac{\partial}{\partial c_{2}}+3 c_{3} \frac{\partial}{\partial c_{3}}\right)\left|I^{(3)}(\alpha, \boldsymbol{c} ; \boldsymbol{\beta})\right\rangle .
\end{aligned}
$$

The relation between the $4 \mathrm{~d}$ and $2 \mathrm{~d}$ parameters is identified by looking at the SeibergWitten curve. Indeed, $T(z)=\sum_{n=-\infty}^{\infty} L_{n} z^{-n-2}$ implies that

$$
\frac{\left\langle 0|T(z)| I^{(3)}(\alpha, \boldsymbol{c} ; \boldsymbol{\beta})\right\rangle}{\left\langle 0 \mid I^{(3)}(\alpha, \boldsymbol{c} ; \boldsymbol{\beta})\right\rangle}=\frac{u}{z^{4}}-\frac{2\left(c_{1} c_{2}+c_{3}(\alpha-2 Q)\right)}{z^{5}}-\frac{c_{2}^{2}+2 c_{3} c_{1}}{z^{6}}-\frac{2 c_{2} c_{3}}{z^{7}}-\frac{c_{3}^{2}}{z^{8}},
$$

where $u \equiv c_{3} \frac{\partial}{\partial c_{1}} \log \left\langle 0 \mid I^{(3)}\left(\alpha, c_{1}, c_{2}, c_{3}\right)\right\rangle-c_{2}(2 \alpha-3 Q)-c_{1}^{2}$. By changing the variable as $z \rightarrow-i c_{3}^{1 / 3} z /\left(1+i c_{2} z / 2 c_{3}^{2 / 3}\right)$, the stress tensor changes as $T \rightarrow-c_{3}^{-2 / 3}\left(1+i c_{2} z / 2 c_{3}^{2 / 3}\right) T .^{24}$

\footnotetext{
${ }^{24}$ Note that this transformation is generated by $L_{0}$ and $L_{-1}$, and therefore the stress tensor transforms as a tensor under it.
} 
Then the above equation is mapped to

$$
\frac{\left\langle 0|T(z)| I^{(3)}(\alpha, \boldsymbol{c} ; \boldsymbol{\beta})\right\rangle}{\left\langle 0 \mid I^{(3)}(\alpha, \boldsymbol{c} ; \boldsymbol{\beta})\right\rangle}=\frac{\widetilde{u}}{z^{4}}+\frac{2 i(2 Q-\alpha)}{z^{5}}+\frac{\frac{\left(c_{2}\right)^{2}}{\left(c_{3}\right)^{4 / 3}}-\frac{4 c_{1}}{\left(c_{3}\right)^{1 / 3}}}{2 z^{6}}+\frac{1}{z^{8}},
$$

where $\widetilde{u} \equiv-u /\left(c_{3}\right)^{2 / 3}+\left(-8 c_{1}\left(c_{2}\right)^{2} c_{3}+\left(c_{2}\right)^{4}+16 c_{2}\left(c_{3}\right)^{2}(\alpha-2 Q)\right) / 16\left(c_{3}\right)^{8 / 3}$. Using (2.8), we can relate this to the Seiberg-Witten curve of the $\left(A_{1}, A_{3}\right)$ theory shown in (2.3). To be concrete, $\widetilde{u}$ is identified with the vev of a Coulomb branch operator of dimension $4 / 3,2 i(2 Q-\alpha)$ is the mass parameter, and $\left(c_{2}\right)^{2} /\left(c_{3}\right)^{4 / 3}-4 c_{1} /\left(c_{3}\right)^{1 / 3}$ is identified with the relevant coupling of dimension $2 / 3$. Note that while the irregular state $\left|I^{(3)}(\alpha, \boldsymbol{c} ; \boldsymbol{\beta})\right\rangle$ depends on $c_{1}, c_{2}$ and $c_{3}$, the Seiberg-Witten curve depends only on the above particular combination of them. This means that there is a redundancy in describing the partition function of the $\left(A_{1}, A_{3}\right)$ theory in terms of the $2 \mathrm{~d}$ irregular conformal block. We use this redundancy to set $c_{1}=0$ in the rest of this section, which turns out to simplify the computation. Under the condition $c_{1}=0$, the $4 \mathrm{~d}$ relevant coupling of dimension $2 / 3$ is identified with

$$
\frac{\left(c_{2}\right)^{2}}{\left(c_{3}\right)^{4 / 3}}=\left(\frac{\left(c_{2}\right)^{3}}{\left(c_{3}\right)^{2}}\right)^{\frac{2}{3}} .
$$

\subsubsection{Irregular conformal block}

Let us now evaluate the rank-three irregular state $\left|I^{(3)}(\alpha, \boldsymbol{c} ; \boldsymbol{\beta})\right\rangle$ explicitly. To that end, we first use the ansatz (2.12) for the rank-three state:

$$
\left|I^{(3)}(\alpha, \boldsymbol{c} ; \boldsymbol{\beta})\right\rangle=c_{2}^{\rho_{2}} c_{3}^{\rho_{3}} e^{\left(\alpha-\beta_{2}\right) S_{3}(\boldsymbol{c})}\left(\left|I^{(2)}\left(\beta_{2}, \widetilde{\boldsymbol{c}} ; \widetilde{\boldsymbol{\beta}}\right)\right\rangle+\sum_{k=1}^{\infty}\left(c_{3}\right)^{k}\left|I_{k}^{(2)}\right\rangle\right),
$$

where $S_{3}(\boldsymbol{c}) \equiv \frac{2 c_{1} c_{2}}{c_{3}}-\frac{c_{2}^{3}}{3 c_{3}^{2}}-\frac{c_{1}^{2}}{c_{2}}, \widetilde{\boldsymbol{c}} \equiv\left(c_{1}, c_{2}\right), \widetilde{\boldsymbol{\beta}} \equiv\left(\beta_{0}, \beta_{1}\right)$, and $\left|I_{k}^{(2)}\right\rangle$ are generalized descendants of the rank-two irregular state $\left|I^{(2)}\left(\beta_{2}, \widetilde{\boldsymbol{c}} ; \widetilde{\boldsymbol{\beta}}\right)\right\rangle{ }^{25}$ The generalized descendants are now of the form

$$
\left(L_{-\ell_{1}}\right)^{p_{1}}\left(L_{-\ell_{2}}\right)^{p_{2}} \cdots\left(L_{-\ell_{n}}\right)^{p_{n}} \frac{\partial^{m_{1}}}{\partial c_{1} m_{1}} \frac{\partial^{m_{2}}}{\partial c_{2} m_{2}}\left|I^{(2)}\left(\beta_{2}, \widetilde{\boldsymbol{c}} ; \widetilde{\boldsymbol{\beta}}\right)\right\rangle
$$

for integers $n, m_{i} \geq 0, p_{i}>0$ and $\ell_{1}>\ell_{2}>\cdots>\ell_{n}>0$. The exponents, $\rho_{2}$ and $\rho_{3}$, are uniquely determined as

$$
\rho_{2}=\frac{1}{2}\left(\beta_{2}-\alpha\right)\left(5 Q-5 \alpha+\beta_{2}\right), \quad \rho_{3}=\frac{2}{3}\left(\alpha-\beta_{2}\right)\left(2 Q-2 \alpha+\beta_{2}\right),
$$

so that the above ansatz solves (3.30)-(3.36). Note that the ansatz (3.40) again fixes the "asymptotic behavior" of $\left|I^{(3)}(\alpha, \boldsymbol{c} ; \boldsymbol{\beta})\right\rangle$ in the limit $c_{3} \rightarrow 0$. The generalized descendants $\left|I_{k}^{(2)}\right\rangle$ are conjectured in [31] to be uniquely determined. We explicitly computed them for $k=1, \cdots, 6$, whose first several expressions are shown in appendix A.2.

\footnotetext{
${ }^{25}$ As mentioned already, our convention for $\left|I_{k}^{(2)}\right\rangle$ is slightly different from that of [31]. Our $\left|I_{k}^{(2)}\right\rangle$ is identical to $\left|I_{3 k}^{(2)}\right\rangle$ of [31].
} 
Using the expressions for the generalized descendants, we see that the inner product of (3.40) and $|0\rangle$ is evaluated as

$$
\left\langle 0 \mid I^{(3)}(\alpha, \boldsymbol{c} ; \boldsymbol{\beta})\right\rangle=\left\langle 0 \mid I^{(2)}\left(\beta_{2}, \widetilde{\boldsymbol{c}} ; \widetilde{\boldsymbol{\beta}}\right)\right\rangle c_{2}^{\rho_{2}} c_{3}^{\rho_{3}} e^{\left(\alpha-\beta_{2}\right) S_{3}(\boldsymbol{c})}\left(\sum_{k=0}^{\infty}\left(\frac{3\left(c_{3}\right)^{2}}{\left(c_{2}\right)^{3}}\right)^{k} D_{k}\left(\alpha, \beta_{2}\right)+\mathcal{O}\left(c_{1}\right)\right),
$$

where

$$
\begin{aligned}
D_{0}=1 & \\
D_{1}=- & \frac{1}{36}\left(\alpha-2 \beta_{2}+Q\right)\left(4 \alpha^{2}+34 \beta_{2}^{2}-34 \beta_{2}(\alpha+Q)+35 \alpha Q-1\right) \\
D_{2}= & \frac{1}{2592}\left(16 \alpha^{6}+\alpha^{4}\left(1801 Q^{2}-200\right)+2 \alpha^{3} Q\left(1365 Q^{2}-1489\right)+50 \alpha Q\left(19-77 Q^{2}\right)\right. \\
& -35 Q^{2}+\alpha^{2}\left(1225 Q^{4}-6244 Q^{2}+169\right)-4 \beta_{2}\left(\alpha-\beta_{2}+Q\right)\left(84 \alpha^{4}-763 \alpha^{2}\right. \\
& +595 \alpha Q^{3}-\beta_{2}\left(\alpha-\beta_{2}+Q\right)\left(561 \alpha^{2}+289 Q^{2}-1156 \beta_{2}\left(\alpha-\beta_{2}+Q\right)+2958 \alpha Q\right. \\
& \left.\left.-2318)+\left(2483 \alpha^{2}-971\right) Q^{2}+\left(1011 \alpha^{2}-3902\right) \alpha Q+271\right)+312 \alpha^{5} Q\right), \\
D_{3}=- & \frac{1}{2} 79936\left(\alpha-2 \beta_{2}+Q\right)\left(64 \alpha^{8}-2352 \alpha^{6}+26796 \alpha^{4}-48313 \alpha^{2}+42875 \alpha^{3} Q^{5}\right. \\
& +1225\left(82 \alpha^{2}-327\right) \alpha^{2} Q^{4}+35\left(2113 \alpha^{4}-19818 \alpha^{2}+33843\right) \alpha Q^{3}+\left(18124 \alpha^{6}\right. \\
& \left.-382215 \alpha^{4}+1420026 \alpha^{2}-5005\right) Q^{2}-2 \beta_{2}\left(\alpha-\beta_{2}+Q\right)\left(944 \alpha^{6}-28008 \alpha^{4}\right. \\
& +243975 \alpha^{2}+62475 \alpha^{2} Q^{4}+70\left(3214 \alpha^{2}-5667\right) \alpha Q^{3}+3\left(40417 \alpha^{4}-382090 \alpha^{2}\right. \\
& +196601) Q^{2}-2 \beta_{2}\left(\alpha-\beta_{2}+Q\right)\left(5100 \alpha^{4}-34 \beta_{2}\left(\alpha-\beta_{2}+Q\right)\left(697 \alpha^{2}+289 Q^{2}\right.\right. \\
& \left.-1156 \beta_{2}\left(\alpha-\beta_{2}+Q\right)+4148 \alpha Q-6852\right)+9 \alpha^{2}\left(21012 Q^{2}-11551\right) \\
& \left.+105 \alpha Q\left(289 Q^{2}-6024\right)-98175 Q^{2}+65841 \alpha^{3} Q+419196\right)+6\left(3212 \alpha^{4}\right. \\
& \left.\left.-66615 \alpha^{2}+256546\right) \alpha Q-224894\right)+\left(1808 \alpha^{6}-55800 \alpha^{4}+517557 \alpha^{2}\right. \\
& -481430) \alpha Q+7560) .
\end{aligned}
$$

The prefactor $\left\langle 0 \mid I^{(2)}\left(\beta_{2}, \widetilde{\boldsymbol{c}} ; \widetilde{\boldsymbol{\beta}}\right)\right\rangle$ in $(3.43)$ is evaluated as follows. Since $\langle 0| L_{1}=\langle 0| L_{0}=$ $0,(3.5)$ and (3.6) imply

$$
\left(c_{2} \frac{\partial}{\partial c_{1}}-2 c_{1}\left(\beta_{2}-Q\right)\right)\left\langle 0 \mid I^{(2)}\left(\beta_{2}, \widetilde{\boldsymbol{c}} ; \widetilde{\boldsymbol{\beta}}\right)\right\rangle=\left(\Delta_{\beta_{2}}+c_{1} \frac{\partial}{\partial c_{1}}+2 c_{2} \frac{\partial}{\partial c_{2}}\right)\left\langle 0 \mid I^{(2)}\left(\beta_{2}, \widetilde{\boldsymbol{c}} ; \widetilde{\boldsymbol{\beta}}\right)\right\rangle=0 .
$$

From this set of differential equations, we see that $\left\langle 0 \mid I^{(2)}\left(\beta_{2}, \widetilde{\boldsymbol{c}}, \widetilde{\boldsymbol{\beta}}\right)\right\rangle$ is given by

$$
\left\langle 0 \mid I^{(2)}\left(\beta_{2}, \widetilde{\boldsymbol{c}}, \widetilde{\boldsymbol{\beta}}\right)\right\rangle=e^{-\left(Q-\beta_{2}\right) \frac{c_{1}^{2}}{c_{2}}} c_{2}^{-\frac{1}{2} \Delta_{\beta_{2}}},
$$

up to a prefactor independent of $c_{1}$ and $c_{2}$. Note here that $\widetilde{\beta}$-dependence only appears in the $c_{i}$-independent prefactor. ${ }^{26}$

\footnotetext{
${ }^{26}$ For this prefactor to be non-vanishing, we need to set $\Delta_{\beta_{0}}=0$. Indeed, $\left|I^{(2)}(\alpha, \boldsymbol{c} ; \boldsymbol{\beta})\right\rangle$ is a linear combination of $\left|I^{(1)}\left(\beta_{1}, c_{1} ; \beta_{0}\right)\right\rangle$ and its generalized descendants, and $\left|I^{(1)}\left(\beta_{1}, c_{1} ; \beta_{0}\right)\right\rangle$ is a linear combination of $\left|\Delta_{\beta_{0}}\right\rangle$ and its Virasoro descendants. Therefore, $\left|I^{(2)}(\alpha, \boldsymbol{c} ; \boldsymbol{\beta})\right\rangle$ is in the Verma module of Virasoro algebra whose highest weight state is $\left|\Delta_{\beta_{0}}\right\rangle$. This means that $\left\langle 0 \mid I^{(2)}(\alpha, \boldsymbol{c} ; \boldsymbol{\beta})\right\rangle=0$ unless $\Delta_{\beta_{0}}=0$.
} 
According to (2.13), the partition function of the $\left(A_{1}, A_{3}\right)$ theory is identified with $\left\langle 0 \mid I^{(3)}(\alpha, \boldsymbol{c} ; \boldsymbol{\beta})\right\rangle$. In this identification, we can set $c_{1}=0$, as discussed at the beginning of this sub-section. Therefore, we have

$$
\mathcal{Z}_{\left(A_{1}, A_{3}\right)}^{\epsilon_{1} \epsilon_{2}}=\left.\left\langle 0 \mid I^{(3)}(\alpha, \boldsymbol{c} ; \boldsymbol{\beta})\right\rangle\right|_{c_{1}=0}=c_{2}^{-\frac{1}{2} \Delta_{\beta_{2}}+\rho_{2}} c_{3}^{\rho_{3}} e^{-\left(\alpha-\beta_{2}\right) \frac{c_{2}^{3}}{3 c_{3}^{2}}} \sum_{k=0}^{\infty}\left(\frac{3 c_{3}^{2}}{c_{2}^{3}}\right)^{k} D_{k}\left(\alpha, \beta_{2}\right),
$$

up to a $c_{i}$-independent prefactor, where the first several coefficients $D_{k}$ are shown in (3.44)(3.47). Recall here that $\left(\left(c_{2}\right)^{3} /\left(c_{3}\right)^{2}\right)^{\frac{2}{3}}$ is identified with the relevant coupling of dimension $2 / 3$ in the $\left(A_{1}, A_{3}\right)$ theory. Therefore (3.50) is an expansion of the $\left(A_{1}, A_{3}\right)$ partition function in the inverse powers of the coupling, i.e., the strong coupling expansion.

Let us now compare the $Q \rightarrow 0$ limit of (3.50) with the strong coupling expansion of the $\left(A_{1}, A_{3}\right)$ partition function evaluated in [15]. ${ }^{27}$ To that end, we first change the variables as

$$
\frac{3\left(c_{3}\right)^{2}}{\left(c_{2}\right)^{3}} \equiv \frac{1}{s}, \quad \alpha \equiv i \theta, \quad \beta_{2} \equiv i\left(\nu+\frac{\theta}{2}\right)
$$

Here, these relations could receive $\mathcal{O}(Q)$ corrections, as we will discuss in section 4 . We omit such $\mathcal{O}(Q)$ corrections for a while since we focus on the $Q \rightarrow 0$ limit here. With the above change of variables, the partition function for $Q=0$ (or equivalently $\epsilon_{1}+\epsilon_{2}=0$ ) is written as

$$
\left.\mathcal{Z}_{\left(A_{1}, A_{3}\right)}^{\epsilon_{1}, \epsilon_{2}}\right|_{\epsilon_{1}+\epsilon_{2}=0}=c_{2}^{-\frac{5}{4} \theta^{2}+2 \theta \nu-\nu^{2}} c_{3}^{\frac{1}{6}(\theta-2 \nu)(3 \theta-2 \nu)} e^{i\left(\nu-\frac{\theta}{2}\right) s} \sum_{k=0}^{\infty} \frac{\left.D_{k}\right|_{Q=0}}{s^{k}},
$$

where

$$
\begin{aligned}
\left.D_{1}\right|_{Q=0}= & -\frac{17}{9} i \nu^{3}+\frac{9 \theta^{2}-2}{36} i \nu, \\
\left.D_{2}\right|_{Q=0}= & -\frac{289}{162} \nu^{6}+\frac{153 \theta^{2}-1159}{324} \nu^{4}-\left(\frac{\theta^{4}}{32}-\frac{11 \theta^{2}}{18}+\frac{271}{648}\right) \nu^{2}-\frac{\theta^{2}\left(11 \theta^{2}-68\right)}{1728}, \\
\left.D_{3}\right|_{Q=0}= & \frac{4913}{4374} i \nu^{9}-\frac{17\left(153 \theta^{2}-2284\right)}{5832} i \nu^{7}+\frac{1377 \theta^{4}-47178 \theta^{2}+279464}{23328} i \nu^{5} \\
& +\frac{-729 \theta^{6}+45648 \theta^{4}-700884 \theta^{2}+899576}{279936} i \nu^{3} \\
& +\frac{-99 \theta^{6}+4270 \theta^{4}-28504 \theta^{2}+3360}{62208} i \nu .
\end{aligned}
$$

We now see that the partition function (3.52) for $Q=0$ coincides, up to a prefactor, with $\mathcal{G}(\nu, s)$ in eq. (3.32) of [15] obtained via the connection to Painlevé II. In particular, our $\left.D_{1}\right|_{Q=0}$ and $\left.D_{2}\right|_{Q=0}$ are precisely identical to $D_{1}$ and $D_{2}$ in eq. (3.33) of [15]. ${ }^{28}$ This

\footnotetext{
${ }^{27}$ In this reference, the $\left(A_{1}, A_{3}\right)$ theory is called the $H_{1}$ Argyres-Douglas theory.

${ }^{28}$ While the $D_{3}$ is not explicitly written in [15], the authors of [37] evaluated it using a nice matrix model description as shown in eq. (3.23) of [37]. We then see that the $Q \rightarrow 0$ limit of our $D_{3}$ is in perfect agreement with the expression.
} 
particularly means that the strong coupling expansion in powers of $1 / s$ corresponds to the asymptotic $c_{3}$-expansion of the rank-3 state $\left|I^{(3)}(\alpha, \boldsymbol{c} ; \boldsymbol{\beta})\right\rangle$.

Note here that (3.53) and (3.54) were also reproduced from the irregular conformal block with one irregular singularity of rank $\frac{3}{2}$ and one regular singularity [26]. On the other hand, we obtained the above results from the irregular conformal block with an irregular singularity of rank 3 without any regular singularity. Therefore, the author of [26] and we computed different irregular conformal blocks of Liouville theory. Nevertheless, the two irregular conformal blocks turn out to be related to the same tau-function of Painlevé II. This is natural from the viewpoint of $4 \mathrm{~d} / 2 \mathrm{~d}$ correspondence. Indeed, the Hitchin systems associated with the two different irregular conformal blocks are isomorhic [68], and therefore these two irregular conformal blocks are expected to be related to the same $4 \mathrm{~d} \mathcal{N}=2 \mathrm{SCFT}$, i.e., the $\left(A_{1}, A_{3}\right)$ theory. Our discussion above explicitly confirms this expectation.

\section{Enhanced flavor symmetries}

Having evaluated the irregular conformal blocks for the $\left(A_{1}, D_{4}\right)$ and $\left(A_{1}, A_{3}\right)$ theories, we here discuss their flavor symmetries. In particular, we will show that the strong coupling expansions of $\mathcal{Z}_{\left(A_{1}, D_{4}\right)}^{\epsilon_{1}, \epsilon_{2}}$ and $\mathcal{Z}_{\left(A_{1}, A_{3}\right)}^{\epsilon_{1}, \epsilon_{2}}$ are invariant under the action of the Weyl group of the enhanced flavor symmetry of the $\mathrm{AD}$ theories.

In general, turning on generic values of mass parameters breaks the flavor symmetry group to its maximal torus. ${ }^{29}$ Therefore the complete flavor symmetry is not visible in the partition function with mass parameters turned on. However, the Weyl group of the flavor symmetry is still a symmetry of the mass-deformed theory, since it is a symmetry of the maximal torus. In particular, the action of the Weyl group permutes the mass parameters in such a way that the partition function is invariant. Below, we identify such an action of the Weyl group for the $\left(A_{1}, D_{4}\right)$ and $\left(A_{1}, A_{3}\right)$ theories.

Furthermore, by demanding this Weyl group symmetry extend to the case of nonvanishing Liouville charge $Q$, we propose an identification of parameters between the Loiuville side and the Argyres-Douglas side for general values of the $\Omega$-background parameters. Under this parameter identification, we find that the $\left(A_{1}, D_{4}\right)$ and $\left(A_{1}, A_{3}\right)$ partition functions are invariant under $Q \rightarrow-Q$, or equivalently $\left(\epsilon_{1}, \epsilon_{2}\right) \rightarrow\left(-\epsilon_{1},-\epsilon_{2}\right)$. We will also give a natural explanation for our parameter identification from the AGT viewpoint, which leads us to a conjecture on the parameter identification for a general rank- $n$ irregular state.

\subsection{Weyl group of the flavor symmetry}

We first study how the Weyl group of the enhanced flavor symmetry is visible in the irregular conformal blocks corresponding to the $\left(A_{1}, D_{4}\right)$ and $\left(A_{1}, A_{3}\right)$ theories.

\footnotetext{
${ }^{29}$ The reason for this is that, in any $4 \mathrm{~d} \mathcal{N}=2$ SCFT, every $\mathcal{N}=2$ preserving mass deformation operator is in the same superconformal multiplet as a flavor current, and therefore in the adjoint representation of the flavor symmetry.
} 


\subsection{1 $\left(A_{1}, D_{4}\right)$ theory}

We start with the $\left(A_{1}, D_{4}\right)$ partition function (3.24) evaluated as the irregular conformal block with one irregular singularity of rank 2 and one regular singularity. The flavor symmetry of the $\left(A_{1}, D_{4}\right)$ theory is enhanced from the manifest $\mathrm{SU}(2) \times \mathrm{U}(1)$ to $\mathrm{SU}(3)$, as reviewed in section 2. Therefore its Weyl group $S_{3}$ is expected to act on the set of mass parameters so that the $\left(A_{1}, D_{4}\right)$ partition function is invariant.

Such an action of $S_{3}$ can easily be found in the limit of $Q \rightarrow 0$. To see this, let us look at the $Q \rightarrow 0$ limit of $\mathcal{Z}_{\left(A_{1}, D_{4}\right)}^{\epsilon_{1}, \epsilon_{2}}$ shown in (3.26). Here, explicit expressions for $\left.D_{k}\right|_{Q=0}$ are shown in (3.27) - (3.28), where $\theta_{s}, \theta_{t}$ and $\nu$ are related by (3.25) to the parameters $\alpha, \beta_{0}$ and $\beta_{1}$ of the corresponding irregular conformal block. As shown in [15], $\theta_{s}$ and $\theta_{t}$ are identified with two mass parameters of the $\left(A_{1}, D_{4}\right)$ theory. We now see that the expansion coefficients $\left.D_{1}\right|_{Q=0}$ and $\left.D_{2}\right|_{Q=0}$ are invariant under both of the following two transformations

$$
\begin{aligned}
& \sigma_{1}: \theta_{s} \longleftrightarrow \theta_{t}, \\
& \sigma_{2}: \theta_{s} \rightarrow \theta_{s}-\theta_{t}, \quad \theta_{t} \rightarrow-\theta_{t} .
\end{aligned}
$$

Note that the above $\sigma_{1}$ and $\sigma_{2}$ satisfy

$$
\sigma_{1}^{2}=\sigma_{2}^{2}=\left(\sigma_{1} \sigma_{2}\right)^{3}=\mathbf{1}
$$

and therefore gives a representation of $S_{3}$. This $S_{3}$ is naturally identified with the Weyl group of the flavor $\mathrm{SU}(3)$ symmetry of $\left(A_{1}, D_{4}\right)$.

Let us now consider the case of $Q \neq 0$. Recall that the Liouville charge $Q$ corresponds to the $\Omega$-background parameters by (2.7). Since the $\Omega$-deformation does not break the flavor symmetry, the above $S_{3}$-invariance of the partition function is expected to extend to the case of $Q \neq 0$. Indeed, we find that $D_{k}$ in (3.24) are invariant under the $S_{3}$ generated by (4.1) and (4.2) if the parameter identification (3.25) receives an $\mathcal{O}(Q)$-correction as

$$
\alpha \equiv i\left(\theta_{s}+\theta_{t}\right)+\frac{3}{2} Q, \quad \beta_{0} \equiv i\left(\theta_{s}-\theta_{t}\right)+\frac{1}{2} Q, \quad \beta_{1}=\frac{i\left(\theta_{s}+\theta_{t}-3 \nu\right)}{3}+\mathcal{O}(Q),
$$

where the $\mathcal{O}(Q)$ correction to $\beta_{1}$ is arbitrary as far as it is independent of $\theta_{s}$ and $\theta_{t} \cdot{ }^{30}$ Note here that the $S_{3}$ invariance requires non-vanishing $\mathcal{O}(Q)$ corrections to the parameter identification (3.25). With the modified identification (4.4), we have checked that $D_{1}, \cdots, D_{4}$ in (3.24) are invariant under the $S_{3}$ generated by (4.1) and (4.2). See appendix B for more detail.

The $\mathcal{O}(Q)$ correction to $\beta_{1}$ is not fixed by the $S_{3}$ invariance. The reason for this is that $\beta_{1}$ is related to the parameter $\nu$ which is identified with the (dual) Coulomb branch parameter $a_{D}$ by $\nu=i a_{D}$ (See the last line of section 4 of [15]). Since $a_{D}$ is neutral under the flavor $\mathrm{SU}(3)$ symmetry, so is $\nu$. Then the flavor symmetry is not enough to fix the $\mathcal{O}(Q)$ correction to the relation between $\beta_{1}$ and $\nu$. Nevertheless, we will conjecture in the next sub-section that the correct identification is $\beta_{1}=i\left(\theta_{s}+\theta_{t}-3 \nu\right) / 3+Q$.

\footnotetext{
${ }^{30}$ The $\mathcal{O}(Q)$-term is expected to be independent of $\theta_{s}$ and $\theta_{t}$, since the $\Omega$-deformation is not coupled with a mass deformation.
} 


\subsection{2 $\left(A_{1}, A_{3}\right)$ theory}

We now turn to the $\left(A_{1}, A_{3}\right)$ partition function evaluated as the irregular conformal block with one irregular singularity of rank 3 . In the limit $Q \rightarrow 0$, the strong coupling expansion of the partition function is given by (3.52). We see that $\left.D_{1}\right|_{Q=0}, \cdots,\left.D_{3}\right|_{Q=0}$ in the expansion are invariant under the $S_{2}$-action

$$
\sigma: \theta \rightarrow-\theta
$$

Since $\theta$ is identified with the mass parameter associated with the flavor $\mathrm{SU}(2)$ symmetry of the $\left(A_{1}, A_{3}\right)$ theory, it is natural to identify this $S_{2}$ as the Weyl group of the flavor symmetry.

This $S_{2}$-invariance of the partition function can be extended to the case of $Q \neq 0$, if the parameter identification (3.51) receives an $\mathcal{O}(Q)$-correction as

$$
\alpha \equiv i \theta+2 Q, \quad \beta_{2}=i\left(\nu+\frac{\theta}{2}\right)+\mathcal{O}(Q)
$$

where the $\mathcal{O}(Q)$ correction to $\beta_{2}$ is arbitrary as far as it is independent of $\theta$. We have checked that $D_{1}, D_{2}$ and $D_{3}$ in (3.45)-(3.47) are invariant under the $S_{2}$-action (4.5), under the modified parameter identification (4.6). Note that the $S_{2}$-invariance of the partition function requires a non-vanishing $\mathcal{O}(Q)$ correction to the parameter identification. The $\mathcal{O}(Q)$ correction to the relation between $\beta_{2}$ and $\nu$ is not fixed by the flavor symmetry, since $\nu$ is related to the (dual) Coulomb branch parameter and therefore neutral under the flavor symmetry. Nevertheless, the correction identification will be conjectured in the next sub-section to be $\beta_{2}=i(\nu+\theta / 2)+3 Q / 2$.

\subsection{Conjectural dictionary between $4 \mathrm{~d}$ and $2 \mathrm{~d}$ parameters}

As seen in the previous sub-section, the strong coupling expansions of the $\left(A_{1}, D_{4}\right)$ and $\left(A_{1}, A_{3}\right)$ partition functions are invariant under the action of the Weyl group of the flavor symmetries. This Weyl group symmetry arises if the parameter identifications, (3.25) and (3.51), are modified by $\mathcal{O}(Q)$ corrections as in (4.4) and (4.6), respectively. This means that there is generally an $\mathcal{O}(Q)$-correction to the relation between the mass parameters of $4 \mathrm{~d} \mathcal{N}=2$ theories and parameters in $2 \mathrm{~d}$ Liouville theory.

To read off a general rule for this $\mathcal{O}(Q)$ correction, note first that $\alpha$ and $\beta_{0}$ in (4.4) are regarded as the Liouville momenta of a rank- 2 state and a rank-0 state, respectively. Indeed, $\alpha$ is the Liouville momentum of $\left|I^{(2)}(\alpha, \boldsymbol{c} ; \boldsymbol{\beta})\right\rangle,{ }^{31}$ and $\beta_{0}$ is the Liouville momentum of the regular primary state $\left|\Delta_{\beta_{0}}\right\rangle$ that arises in the expansion of $\left|I^{(2)}(\alpha, \boldsymbol{c} ; \boldsymbol{\beta})\right\rangle$ in terms of generalized descendants. ${ }^{32}$ Similarly, $\alpha$ in (4.6) is the Liouville momentum of the rank-3 state $\left|I^{(3)}(\alpha, \boldsymbol{c} ; \boldsymbol{\beta})\right\rangle$. More generally, as reviewed in section 2 , the parameters $\alpha$ and $\beta_{k}$ of $\left|I^{(n)}(\alpha, \boldsymbol{c} ; \boldsymbol{\beta})\right\rangle$ are respectively regarded as the Liouville momenta of rank- $n$ and rank- $k$ states, in the expansion in terms of generalized descendants. This follows from the general ansatz (2.12) for $\left|I^{(n)}(\alpha, \boldsymbol{c} ; \boldsymbol{\beta})\right\rangle$ proposed in [31].

\footnotetext{
${ }^{31}$ Recall here that we call $\alpha$ in (2.9) the "Liouville momentum" of the irregular state of rank $n$.

${ }^{32}$ Recall that a rank-zero state is identified as a regular primary state.
} 
With this correspondence in mind, we now see that the first two equations of (4.4) and the first equation of (4.6) satisfy the following rule: when the Liouville momentum $\alpha$ of a rank-n state is associated with a four-dimensional mass parameter (or the vev of a Coulomb branch parameter) $m$, the precise relation between them is given by

$$
\alpha=m+\frac{n+1}{2} Q .
$$

Here $m$ is a mass parameter or the vev of a (dual) Coulomb branch operator, depending on whether the corresponding symmetry is gauged.

Note that the above rule (4.7) is a natural generalization of the original AGT correspondence involving only regular primary states [28]. In [28], it was shown that the Liouville momentum $\gamma$ of a regular primary state is related to a mass parameter $m$ of the four-dimensional theory by $\gamma=m+Q / 2$. Since the regular state is regarded as the rank-zero state, this is a special case of (4.7). The factor $\frac{n+1}{2}$ in (4.7) is understood as follows. Recall that the irregular state of rank $n$ is constructed by taking a colliding limit of $(n+1)$ regular primary states. As reviewed in section 2, the Liouville momentum $\alpha$ of the resulting rank- $n$ state $\left|I^{(n)}(\alpha, \boldsymbol{c} ; \boldsymbol{\beta})\right\rangle$ is the sum of the Liouville momenta of the original $(n+1)$ regular primary states, say $\gamma_{0}, \cdots, \gamma_{n}$. On the other hand, the original AGT correspondence implies that the $i$-th momentum $\gamma_{i}$ is related to a four-dimensional mass parameter $m_{i}$ by $\gamma_{i}=m_{i}+Q / 2$. Combining these two, we see that

$$
\alpha=\sum_{i=0}^{n} \gamma_{i}=\left(\sum_{i=0}^{n} m_{i}\right)+\frac{n+1}{2} Q .
$$

By identifying $\sum_{i=0}^{n} m_{i}$ as the $4 \mathrm{~d}$ mass parameter $m$ corresponding to the Liouville momentum $\alpha$, we see that (4.8) is precisely identical to the rule (4.7).

Given the above interpretation of (4.7), we now conjecture that the rule (4.7) generally applies to the Liouville momentum $\alpha$ of any Liouville irregular state of integer rank $n$ and the corresponding $4 \mathrm{~d}$ mass parameter $m .{ }^{33}$ This particularly implies that the $\mathcal{O}(Q)$ correction to $\beta_{1}$ in (4.4) is fixed by

$$
\beta_{1}=\frac{i\left(\theta_{s}+\theta_{t}-3 \nu\right)}{3}+Q
$$

and that to $\beta_{2}$ in (4.6) is fixed by

$$
\beta_{2}=i\left(\nu+\frac{\theta}{2}\right)+\frac{3}{2} Q
$$

Below, we rewrite the strong coupling expansions of the $\left(A_{1}, D_{4}\right)$ and $\left(A_{1}, A_{3}\right)$ partition functions using (4.4) and (4.6) restricted by (4.9) and (4.10).

\footnotetext{
${ }^{33}$ There are also irregular states of half-integer rank. They are not constructed in a colliding limit of regular primary states, and moreover they have no parameter corresponding to a $4 \mathrm{~d}$ mass parameter.
} 


\subsection{1 $\left(A_{1}, D_{4}\right)$ partition function}

We first study the $\left(A_{1}, D_{4}\right)$ partition function. Using (4.4) restricted by (4.9), we see that the coefficients $D_{i}$ in the strong coupling expansion (3.24) are rewritten as

$$
\begin{aligned}
D_{1}= & \left(3 \nu^{3}-2 \nu\left(\theta_{s}^{2}-\theta_{s} \theta_{t}+\theta_{t}^{2}\right)-\frac{2}{9}\left(2 \theta_{s}-\theta_{t}\right)\left(\theta_{s}+\theta_{t}\right)\left(\theta_{s}-2 \theta_{t}\right)\right)-\frac{3 \nu}{4} Q^{2} \\
D_{2}= & \frac{9 \nu^{6}}{2}+\nu^{4}\left(\frac{105}{16}-6\left(\theta_{s}^{2}-\theta_{s} \theta_{t}+\theta_{t}^{2}\right)\right)-\frac{2}{3} \nu^{3}\left(\theta_{s}-2 \theta_{t}\right)\left(2 \theta_{s}-\theta_{t}\right)\left(\theta_{s}+\theta_{t}\right) \\
& +\nu^{2}\left(\frac{1}{2}\left(\theta_{s}^{2}-\theta_{s} \theta_{t}+\theta_{t}^{2}\right)\left(4\left(\theta_{s}^{2}-\theta_{s} \theta_{t}+\theta_{t}^{2}\right)-11\right)+\frac{3}{16}\right) \\
& +\frac{\nu}{9}\left(2 \theta_{s}-\theta_{t}\right)\left(\theta_{s}+\theta_{t}\right)\left(\theta_{s}-2 \theta_{t}\right)\left(4\left(\theta_{s}^{2}-\theta_{s} \theta_{t}+\theta_{t}^{2}\right)-7\right) \\
+ & \frac{1}{324}\left(32 \theta_{s}^{6}-96 \theta_{s}^{5} \theta_{t}+\theta_{s}^{4}\left(36-24 \theta_{t}^{2}\right)+8 \theta_{s}^{3} \theta_{t}\left(26 \theta_{t}^{2}-9\right)-3 \theta_{s}^{2}\left(8 \theta_{t}^{4}-36 \theta_{t}^{2}+9\right)\right. \\
& \left.\quad-3 \theta_{s} \theta_{t}\left(32 \theta_{t}^{4}+24 \theta_{t}^{2}-9\right)+\theta_{t}^{2}\left(32 \theta_{t}^{4}+36 \theta_{t}^{2}-27\right)\right) \\
& +\frac{1}{192}\left(-432 \nu^{4}+18 \nu^{2}\left(16\left(\theta_{s}^{2}-\theta_{s} \theta_{t}+\theta_{t}^{2}\right)-37\right)+32 \nu\left(2 \theta_{s}-\theta_{t}\right)\left(\theta_{s}+\theta_{t}\right)\left(\theta_{s}-2 \theta_{t}\right)\right. \\
& \left.\quad+104\left(\theta_{s}^{2}-\theta_{s} \theta_{t}+\theta_{t}^{2}\right)-3\right) Q^{2}+\frac{1}{256}\left(72 \nu^{2}+25\right) Q^{4} .
\end{aligned}
$$

Note that they are invariant under the action of $S_{3}$ generated by (4.1) and (4.2), as expected. Moreover, they are also invariant under $Q \rightarrow-Q$, or equivalently $\left(\epsilon_{1}, \epsilon_{2}\right) \rightarrow$ $\left(-\epsilon_{1},-\epsilon_{2}\right)$. We have checked this invariance up to $D_{4}$. We will briefly comment on this symmetry in the next section.

\subsection{2 $\left(A_{1}, A_{3}\right)$ partition function}

Let us turn to the $\left(A_{1}, A_{3}\right)$ partition function. Using (4.6) restricted by (4.10), the coefficients $D_{k}$ in the strong coupling expansion (3.50) are rewritten as

$$
\begin{aligned}
D_{1}= & -\frac{i \nu\left(68 \nu^{2}-9 \theta^{2}+2\right)}{36}+\frac{19}{36} i \nu Q^{2} \\
D_{2}= & -\frac{289}{162} \nu^{6}+\frac{153 \theta^{2}-1159}{324} \nu^{4}-\left(\frac{\theta^{4}}{32}-\frac{11 \theta^{2}}{18}+\frac{271}{648}\right) \nu^{2}-\frac{\theta^{2}\left(11 \theta^{2}-68\right)}{1728} \\
& +\left(\frac{323}{324} \nu^{4}-\left(\frac{19 \theta^{2}}{144}-\frac{349}{162}\right) \nu^{2}-\frac{71 \theta^{2}}{864}+\frac{17}{432}\right) Q^{2}-\left(\frac{361}{2592} \nu^{2}+\frac{131}{1728}\right) Q^{4} \\
D_{3}= & \frac{413 i}{4374} \nu^{9}-\frac{17 i\left(153 \theta^{2}-2284\right)}{5832} \nu^{7}+\frac{i\left(1377 \theta^{4}-47178 \theta^{2}+279464\right)}{23328} \nu^{5} \\
& -\frac{i\left(9 \theta^{2}\left(81 \theta^{4}-5072 \theta^{2}+77876\right)-899576\right)}{279936} \nu^{3}-\frac{i\left(99 \theta^{6}-4270 \theta^{4}+28504 \theta^{2}-3360\right)}{62208} \nu \\
& +\left(-\frac{5491 i \nu^{7}}{5832}+\frac{i\left(2907 \theta^{2}-68839\right)}{11664} \nu^{5}-\frac{i\left(1539 \theta^{4}-94152 \theta^{2}+1161988\right)}{93312} \nu^{3}\right. \\
& \left.-\frac{i\left(1487 \theta^{4}-61300 \theta^{2}+66264\right)}{62208} \nu\right) Q^{2}+\left(\frac{6137 i}{23328} \nu^{5}-\frac{i\left(3249 \theta^{2}-118736\right)}{93312} \nu^{3}\right. \\
& \left.-\frac{i\left(3877 \theta^{2}-92390\right) \nu}{62208}\right) Q^{4}+\left(-\frac{6859 i \nu^{3}}{279936}-\frac{2489 i \nu}{62208}\right) Q^{6}
\end{aligned}
$$


These coefficients are invariant under the $S_{2}$-action (4.5), as expected. Moreover, we see that they are also invariant under $Q \rightarrow-Q$, or equivalently $\left(\epsilon_{1}, \epsilon_{2}\right) \rightarrow\left(-\epsilon_{1},-\epsilon_{2}\right)$. We will briefly comment on this symmetry in the next section.

\section{Conclusions and discussions}

In this paper, we have evaluated Liouville irregular states of rank-two and three, based on an ansatz proposed in [31]. Using these two irregular states, we have computed Liouville irregular conformal blocks corresponding to the partition functions of the $\left(A_{1}, A_{3}\right)$ and $\left(A_{1}, D_{4}\right)$ theories for general $\Omega$-background parameters $\epsilon_{1}$ and $\epsilon_{2}$. In the limit $\epsilon_{1}+\epsilon_{2} \rightarrow 0$, our result correctly reproduces the strong coupling expansions of the partition functions obtained from the tau-functions of Painlevé II and IV. This confirms that the ansatz proposed in [31] gives consistent results with [24-26]. Moreover, we have shown that the asymptotic expansion of an irregular state proposed in [31] corresponds to the strong coupling expansion of partition functions studied in [15]. In addition, our result on $\left(A_{1}, A_{3}\right)$ implies that the irregular conformal block with one irregular singularity of rank 3 is also related to the tau-function of Painlevé II, in a similar way as that with one irregular singularity of rank $\frac{3}{2}$ and a regular singularity [26].

We have also shown that our partition functions are invariant under the action of the Weyl group of the flavor symmetry when the Liouville momentum of irregular states is appropriately identified with the linear combination of a mass parameter and the $\Omega$ background parameters. From this observation, we have conjectured a general relation (4.7) between the Liouville momentum of rank- $n$ irregular state and the corresponding mass parameter in four dimensions. We have also given an interpretation of this conjectured relation in terms of the colliding limit of regular singularities.

With the conjectured relation (4.7), we find that the partition functions $\mathcal{Z}_{\left(A_{1}, A_{3}\right)}^{\epsilon_{1}, \epsilon_{2}}$ and $\mathcal{Z}_{\left(A_{1}, D_{4}\right)}^{\epsilon_{1}, \epsilon_{2}}$ are invariant under $\left(\epsilon_{1}, \epsilon_{2}\right) \rightarrow\left(-\epsilon_{1},-\epsilon_{2}\right)$ with masses and the vev of Coulomb branch operators fixed. This invariance is consistent with quantum periods of ArgyresDouglas theories recently evaluated in $[22,23,73]$. The quantum periods are $a_{I} \equiv \oint_{A_{I}} \lambda$ and $a_{D I} \equiv \oint_{B_{I}} \lambda$ deformed by the $\Omega$-background $\left(\epsilon_{1}, \epsilon_{2}\right)=(\hbar, 0)$, where $A_{I}$ and $B_{I}$ are canonical 1-cycles of the Seiberg-Witten curve, and $\lambda$ is the $\Omega$-deformed Seiberg-Witten 1-form. These periods are related to the deformed prepotential $\mathcal{F} \equiv \lim _{\left(\epsilon_{1}, \epsilon_{2}\right) \rightarrow(\hbar, 0)}\left(-\epsilon_{1} \epsilon_{2} \log \mathcal{Z}^{\epsilon_{1}, \epsilon_{2}}\right)$ by $a_{D}=\partial \mathcal{F} / \partial a$. As shown in $[22,23]$, the $\hbar$-expansion of these quantum periods for $\left(A_{1}, A_{r}\right)$ and $\left(A_{1}, D_{r}\right)$ theories have only even powers of $\hbar^{34}$ This means that, at least in the limit of $\epsilon_{2} \rightarrow 0$, the quantity $\epsilon_{1} \epsilon_{2} \log \mathcal{Z}^{\epsilon_{1}, \epsilon_{2}}$ is invariant under $\left(\epsilon_{1}, \epsilon_{2}\right) \rightarrow\left(-\epsilon_{1},-\epsilon_{2}\right)$. Our result suggests that this invariance extends to the case of $\epsilon_{1}, \epsilon_{2} \neq 0$. We leave a detailed study of this symmetry for future work.

\footnotetext{
${ }^{34}$ In [22], the $\left(A_{1}, A_{3}\right)$ and $\left(A_{1}, D_{4}\right)$ theories are realized at the most singular point on the Coulomb branch of $\mathrm{SU}(2)$ gauge theory with two and three flavors, respectively. Its generalization to the whole $\left(A_{1}, A_{r}\right)$ and $\left(A_{1}, D_{r}\right)$ theories was carefully studied in [23]. See [73] for discussions around the monopole point on the Coulomb branch.
} 


\section{Acknowledgments}

The authors thank Katsushi Ito, Yusuke Kimura, Kazunobu Maruyoshi, Takafumi Okubo, Makoto Sakaguchi, Sakura Schäfer-Nameki and Yuji Sugawara for various discussions. T.N. particularly thanks Matthew Buican for various illuminating dicussions in many collaborations on related topics. The authors also thank the organizers of the international workshop "KEK Theory workshop 2018," where they had many useful discussions on this topic. The work of T.N. is partially supported by JSPS Grant-in-Aid for Early-Career Scientists 18K13547. The work of T.U. is supported by Grant-in-Aid for JSPS Research Fellows 19J11212.

\section{A Computations of generalized descendants}

In this appendix, we explain a little more about the computations of generalized descendants involved in irregular states of rank-two and three, following [31].

\section{A.1 Rank-two state $\left|I^{(2)}(\alpha, c ; \beta)\right\rangle$}

Let us start with the rank-two irregular state $\left|I^{(2)}(\alpha, \boldsymbol{c} ; \boldsymbol{\beta})\right\rangle$, where $\boldsymbol{c}=\left(c_{1}, c_{2}\right)$ and $\boldsymbol{\beta}=$ $\left(\beta_{0}, \beta_{1}\right)$. According to [31], this state is expected to be expanded as (3.9) in terms of generalized descendants $\left|I_{k}^{(1)}\right\rangle$ for $k=1,2, \cdots$. These states are fixed order by order so that (3.9) satisfies (3.2)-(3.6). Indeed, substituting (3.9) in (3.2)-(3.6) leads to the following equations for each $k$ :

$$
\begin{aligned}
L_{n>4}\left|I_{k}^{(1)}\right\rangle & =0, \\
L_{4}\left|I_{k}^{(1)}\right\rangle & =-\left|I_{k-2}^{(1)}\right\rangle, \\
L_{3}\left|I_{k}^{(1)}\right\rangle & =-2 c_{1}\left|I_{k-1}^{(1)}\right\rangle, \\
\left(L_{2}+c_{1}^{2}\right)\left|I_{k}^{(1)}\right\rangle & =-(2 \alpha-3 Q)\left|I_{k-1}^{(1)}\right\rangle, \\
\left(L_{1}+2 c_{1}(\beta-Q)\right)\left|I_{k}^{(1)}\right\rangle & =\left(\frac{\partial}{\partial c_{1}}+\frac{\nu_{1}}{c_{1}}\right)\left|I_{k-1}^{(1)}\right\rangle, \\
L_{0}\left|I_{k}^{(1)}\right\rangle & =\left(\Delta_{\beta}+2 k+c_{1} \frac{\partial}{\partial c_{1}}\right)\left|I_{k}^{(1)}\right\rangle .
\end{aligned}
$$

Solving these equations successively for $k=1,2, \cdots$, we obtain $\left|I_{k}^{(1)}\right\rangle$ for all $k$. To be concrete, in evaluating $\left|I_{k}^{(1)}\right\rangle$, we write down the most general linear combination of level$2 k$ generalized desendants, and then fix their coefficients by demanding that the equations (A.1)-(A.5) are satisfied. Note that $L_{-1}, 1 / c_{1}$ and $\partial_{c_{1}}$ are all regarded as level-one. The results for $k=1$ and $k=2$ are shown in (3.13) and (3.14). In appendix B, we show the coefficients $D_{k}$ appearing in (3.18) for $k=1, \cdots, 4$. For computing $D_{k}$ for $k=1, \cdots, 4$, we need to evaluate $\left|I_{k}^{(1)}\right\rangle$ for $k=1, \cdots, 5$.

\section{A.2 Rank-three state $\left|I^{(3)}(\alpha, \boldsymbol{c} ; \boldsymbol{\beta})\right\rangle$}

Let us turn to the rank-three irregular state $\left|I^{(3)}(\alpha, \boldsymbol{c} ; \boldsymbol{\beta})\right\rangle$, where $\boldsymbol{c}=\left(c_{1}, c_{2}, c_{3}\right)$ and $\beta=\left(\beta_{0}, \beta_{1}, \beta_{2}\right)$. As in section A.1, we fix the generalized descendants $\left|I_{k}^{(2)}\right\rangle$ in the 
ansatz (3.40) by demanding that (3.40) satisfies (3.30)-(3.36). To that end, we substitute (3.40) in (3.30)-(3.36) to obtain

$$
\begin{aligned}
L_{n>6}\left|I_{k}^{(2)}\right\rangle & =0 \\
L_{6}\left|I_{k}^{(2)}\right\rangle & =-\left|I_{k-2}^{(2)}\right\rangle \\
L_{5}\left|I_{k}^{(2)}\right\rangle & =-2 c_{2}\left|I_{k-2}^{(2)}\right\rangle, \\
\left(L_{4}+c_{2}^{2}\right)\left|I_{k}^{(2)}\right\rangle & =-2 c_{1}\left|I_{k-1}^{(2)}\right\rangle, \\
\left(L_{3}+2 c_{1} c_{2}\right)\left|I_{k}^{(2)}\right\rangle & =-2(\alpha-2 Q)\left|I_{k-1}^{(2)}\right\rangle, \\
\left(L_{2}+c_{2}(2 \beta-3 Q)+c_{1}^{2}\right)\left|I_{k}^{(2)}\right\rangle & =\left(\partial_{c_{1}}-\frac{2 c_{1}}{c_{2}}(\alpha-\beta)\right)\left|I_{k-1}^{(2)}\right\rangle, \\
\left(L_{1}+2 c_{1}(\beta-Q)-c_{2} \partial_{c_{1}}\right)\left|I_{k}^{(2)}\right\rangle & =\left(2 \partial_{c_{2}}+\frac{2 c_{1}^{2}}{c_{2}^{2}}(\alpha-\beta)+\frac{2 \rho_{2}}{c_{2}}\right)\left|I_{k-1}^{(2)}\right\rangle, \\
L_{0}\left|I_{k}^{(2)}\right\rangle & =\left(\Delta_{\beta}+3 k+c_{1} \partial_{c_{1}}+2 c_{2} \partial_{c_{2}}\right)\left|I_{k}^{(2)}\right\rangle .
\end{aligned}
$$

We solve these equations successively for $k=1,2, \cdots$. The strategy is again to write down the most general linear combination of level-3k generalized descendants for $\left|I_{k}^{(2)}\right\rangle$, and fix their coefficients so that the above equations are satisfied. Note here that $L_{-1}, 1 / c_{1}$ and $\partial_{c_{1}}$ are regarded as level-one, and $1 / c_{2}$ and $\partial_{c_{2}}$ are regarded as level-two.

As discussed in the 3.2, we only need to evaluate the leading contributions in the $c_{1} \rightarrow 0$ limit. Below, we write down expressions for the first several $\left|I_{k}^{(2)}\right\rangle$ :

$$
\begin{aligned}
\left|I_{0}^{(2)}\right\rangle= & \left|I^{(2)}\left(\beta, c_{1}, c_{2}\right)\right\rangle \\
\left|I_{1}^{(2)}\right\rangle= & \left(\frac{1}{3 c_{2}} L_{-1}+\frac{3 \alpha-4 \beta}{3 c_{2}} \partial_{c_{1}}-\frac{2 c_{1}}{3 c_{2}} \partial_{c_{2}}\right. \\
& \left.\quad-\frac{2\left(\rho_{2}-\beta^{2}+\beta \alpha+Q(\beta-\alpha)\right) c_{1}}{c_{2}^{2}}+\frac{2(\beta-\alpha) c_{1}^{3}}{3 c_{2}^{3}}\right)\left|I^{(2)}\left(\beta, c_{1}, c_{2}\right)\right\rangle, \\
\left|I_{2}^{(2)}\right\rangle= & \left(\frac{\rho_{4}}{c_{2}^{3}}-\frac{1}{6 c_{2}^{2}} L_{-2}+\frac{\frac{Q}{6}+\frac{19}{9} \beta-\frac{5 \alpha}{3}}{c_{2}^{2}} \partial_{c_{2}}+\frac{1}{18 c_{2}^{2}} L_{-1}^{2}+\frac{(-4 \beta+3 \alpha)}{9 c_{2}^{2}} L_{-1} \partial_{c_{1}}\right. \\
& \left.+\frac{-3+64 \beta^{2}-96 \beta \alpha+36 \alpha^{2}}{72 c_{2}^{2}} \partial_{c_{1}}^{2}+\mathcal{O}\left(c_{1}\right)\right)\left|I^{(2)}\left(\beta, c_{1}, c_{2}\right)\right\rangle,
\end{aligned}
$$

where $\rho_{4}$ is determined as

$$
\rho_{4}=\frac{1}{12}\left(\beta_{3}-\alpha\right)\left(4 \alpha^{2}-26 \alpha \beta_{3}+34 \beta_{3}^{2}+35 Q^{2}+27 \alpha Q-69 \beta_{3} Q-1\right) .
$$

In section 3.2, we write down expressions for $D_{1}, \cdots, D_{3}$ appearing in the expansion (3.43). To evaluate these three coefficients, we need to evaluate $\left|I_{k}^{(2)}\right\rangle$ for $k=1, \cdots, 6$, up to subleading terms in the limit $c_{1} \rightarrow 0$.

\section{B More coefficients for the $\left(A_{1}, D_{4}\right)$ partition function}

We here show the coefficients $D_{i}$ in (3.26) up to $i=4$ using (4.4) and (4.9). These coefficients are written in terms of symmetric polynomials in the variables $X_{1}, X_{2}$ and $X_{3}$, 
where

$$
\begin{aligned}
& X_{1}=\theta_{s}-2 \theta_{t}, \\
& X_{2}=\theta_{t}-2 \theta_{s}, \\
& X_{3}=\theta_{s}+\theta_{t} .
\end{aligned}
$$

This reflects the fact that the mass parameters are permuted by the action of the Weyl group of the flavor $\mathrm{SU}(3)$ symmetry of the $\left(A_{1}, D_{4}\right)$ theory, as discussed in section 4 . Indeed, these three variables are permuted under the $S_{3}$ Weyl transformations (4.1) and (4.2). The elementary symmetric polynomials $s_{1}, s_{2}$ and $s_{3}$ of these variables are given by

$$
\begin{aligned}
& s_{1}=X_{1}+X_{2}+X_{3}=0, \\
& s_{2}=X_{1} X_{2}+X_{2} X_{3}+X_{3} X_{1}=-3\left(\theta_{s}^{2}-\theta_{s} \theta_{t}+\theta_{t}^{2}\right), \\
& s_{3}=X_{1} X_{2} X_{3}=\left(\theta_{s}-2 \theta_{t}\right)\left(\theta_{t}-2 \theta_{s}\right)\left(\theta_{s}+\theta_{t}\right) .
\end{aligned}
$$

Since the partition function is supposed to be invariant under the action of the Weyl group, the coefficients $D_{i}$ of its strong coupling expansion are expected to be written in terms of $s_{2}$ and $s_{3}$. Indeed, using (B.2), we may rewrite $D_{1}$ and $D_{2}$ as

$$
\begin{aligned}
D_{1}= & 3 \nu^{3}+\frac{2 \nu s_{2}}{3}+\frac{2 s_{3}}{9}-\frac{3 \nu}{4} Q^{2} \\
D_{2}= & \frac{9 \nu^{6}}{2}+\nu^{4}\left(2 s_{2}+\frac{105}{16}\right)+\frac{2 \nu^{3} s_{3}}{3}+\frac{\nu^{2}}{144}\left(8 s_{2}\left(4 s_{2}+33\right)+27\right)+\frac{\nu}{27}\left(4 s_{2}+21\right) s_{3} \\
& +\frac{1}{324}\left(s_{2}\left(4 s_{2}+9\right)+8 s_{3}^{2}\right) \\
& +\left(-\frac{9 \nu^{4}}{4}+\nu^{2}\left(-\frac{s_{2}}{2}-\frac{111}{32}\right)-\frac{\nu s_{3}}{6}+\left(-\frac{13 s_{2}}{72}-\frac{1}{64}\right)\right) Q^{2} \\
& +\left(\frac{9 \nu^{2}}{32}+\frac{25}{256}\right) Q^{4} .
\end{aligned}
$$

Moreover, $D_{3}$ and $D_{4}$ are also evaluated as

$$
\begin{aligned}
D_{3}= & -\frac{9 \nu^{9}}{2}+\nu^{7}\left(-3 s_{2}-\frac{315}{16}\right)-\nu^{6} s_{3}+\nu^{5}\left(-\frac{2 s_{2}^{2}}{3}-\frac{79 s_{2}}{8}-\frac{411}{16}\right)-\frac{\nu^{4}}{72}\left(32 s_{2}+273\right) s_{3} \\
& +\frac{\nu^{3}}{648}\left(-32 s_{2}^{3}-816 s_{2}^{2}-5535 s_{2}-48 s_{3}^{2}-1701\right)-\frac{\nu^{2}}{648}\left(32 s_{2}^{2}+600 s_{2}+2475\right) s_{3} \\
& +\frac{\nu}{486}\left(-4 s_{2}^{3}-105 s_{2}^{2}-s_{2}\left(8 s_{3}^{2}+297\right)-84 s_{3}^{2}\right)-\frac{1}{4374} s_{3}\left(12 s_{2}^{2}+351 s_{2}+8 s_{3}^{2}+972\right) \\
+ & \left(\frac{27 \nu^{7}}{8}+\nu^{5}\left(\frac{3 s_{2}}{2}+\frac{981}{64}\right)+\frac{\nu^{4} s_{3}}{2}+\frac{1}{48} \nu^{3}\left(8 s_{2}^{2}+203 s_{2}+1134\right)+\nu^{2}\left(\frac{s_{2}}{9}+\frac{65}{48}\right)\right. \\
& \left.\left.\quad+\frac{1}{864} \nu\left(112 s_{2}^{2}+2307 s_{2}+16 s_{3}^{2}+621\right)\right) s_{3}+\frac{1}{2592}\left(104 s_{2}+1305\right) s_{3}\right) Q^{2} \\
+ & \left(-\frac{27 \nu^{5}}{32}-\frac{3}{256} \nu^{3}\left(16 s_{2}+247\right)-\frac{\nu^{2} s_{3}}{16}+\frac{\nu}{768}\left(-154 s_{2}-1695\right)-\frac{25 s_{3}}{1152}\right) Q^{4} \\
+ & \left(\frac{9 \nu^{3}}{128}+\frac{75 \nu}{1024}\right) Q^{6},
\end{aligned}
$$




$$
\begin{aligned}
& D_{4}=-\frac{27 \nu^{12}}{8}+\nu^{10}\left(-3 s_{2}-\frac{945}{32}\right)-\nu^{9} s_{3}+\nu^{8}\left(-s_{2}^{2}-\frac{171 s_{2}}{8}-\frac{50049}{512}\right)-\frac{\nu^{7}}{24}\left(16 s_{2}+189\right) s_{3} \\
& +\nu^{6}\left(-\frac{4 s_{2}^{3}}{27}-\frac{373 s_{2}^{2}}{72}-\frac{1737 s_{2}}{32}-\frac{s_{3}^{2}}{9}-\frac{34335}{256}\right)-\frac{\nu^{5}}{432}\left(64 s_{2}^{2}+1620 s_{2}+9567\right) s_{3} \\
& +\nu^{4}\left(-\frac{2 s_{2}^{4}}{243}-\frac{35 s_{2}^{3}}{81}-\frac{1153 s_{2}^{2}}{144}+\left(-\frac{4 s_{3}^{2}}{81}-\frac{3323}{64}\right) s_{2}-\frac{49 s_{3}^{2}}{72}-\frac{15381}{512}\right) \\
& -\frac{\nu^{3}}{11664} s_{3}\left(128 s_{2}^{3}+5280 s_{2}^{2}+70740 s_{2}+64 s_{3}^{2}+273861\right) \\
& +\frac{\nu^{2}}{93312}\left(-256 s_{2}^{4}-14976 s_{2}^{3}-8\left(64 s_{3}^{2}+30969\right) s_{2}^{2}-18\left(832 s_{3}^{2}+45063\right) s_{2}\right. \\
& \left.-9\left(11888 s_{3}^{2}+5103\right)\right) \\
& -\frac{\nu}{52488} s_{3}\left(96 s_{2}^{3}+5616 s_{2}^{2}+64 s_{3}^{2} s_{2}+77274 s_{2}+1008 s_{3}^{2}+207765\right) \\
& +\frac{1}{629856}\left(-48 s_{2}^{4}-2808 s_{2}^{3}-3\left(64 s_{3}^{2}+8829\right) s_{2}^{2}-27\left(400 s_{3}^{2}+1701\right) s_{2}\right. \\
& \left.-16 s_{3}^{2}\left(4 s_{3}^{2}+4617\right)\right) \\
& +\left(\frac{27 \nu^{10}}{8}+\frac{9 \nu^{8}}{8}\left(2 s_{2}+27\right)+\frac{3 s_{3} \nu^{7}}{4}+\frac{\nu^{6}}{512}\left(256 s_{2}^{2}+7760 s_{2}+57555\right)+\frac{\nu^{5}}{96}\left(32 s_{2}+495\right) s_{3}\right. \\
& +\nu^{4}\left(\frac{s_{2}^{3}}{27}+\frac{299 s_{2}^{2}}{144}+\frac{4803 s_{2}}{128}+\frac{s_{3}^{2}}{18}+\frac{193455}{1024}\right)+\frac{\nu^{3}}{864}\left(32 s_{2}^{2}+1148 s_{2}+10611\right) s_{3} \\
& +\frac{\nu^{2}}{82944}\left(3840 s_{2}^{3}+190656 s_{2}^{2}+8\left(128 s_{3}^{2}+338931\right) s_{2}+9\left(1984 s_{3}^{2}+168183\right)\right) \\
& +\frac{\nu}{46656} s_{3}\left(1344 s_{2}^{2}+52380 s_{2}+64 s_{3}^{2}+426951\right) \\
& \left.+\frac{1}{373248}\left(832 s_{2}^{3}+85752 s_{2}^{2}+1664 s_{3}^{2} s_{2}+387018 s_{2}+41616 s_{3}^{2}+15309\right)\right) Q^{2} \\
& +\left(-\frac{81 \nu^{8}}{64}-\frac{9 \nu^{6}}{256}\left(16 s_{2}+287\right)-\frac{3 \nu^{5} s_{3}}{16}+\frac{\nu^{4}}{4096}\left(-256 s_{2}^{2}-11680 s_{2}-126603\right)\right. \\
& -\frac{\nu^{3}}{384}\left(16 s_{2}+331\right) s_{3}+\frac{\nu^{2}}{36864}\left(-4256 s_{2}^{2}-157176 s_{2}-256 s_{3}^{2}-1416717\right) \\
& \left.-\frac{\nu}{6912}\left(308 s_{2}+6507\right) s_{3}+\frac{1}{663552}\left(-11616 s_{2}^{2}-873720 s_{2}-1600 s_{3}^{2}-378189\right)\right) Q^{4} \\
& +\left(\frac{27 \nu^{6}}{128}+\frac{3 \nu^{4}}{128}\left(2 s_{2}+51\right)+\frac{\nu^{3} s_{3}}{64}+\frac{3 \nu^{2}}{8192}\left(272 s_{2}+5433\right)\right. \\
& \left.+\frac{25 \nu s_{3}}{1536}+\frac{1}{147456}\left(2600 s_{2}+98613\right)\right) Q^{6} \\
& +\left(-\frac{27 \nu^{4}}{2048}-\frac{225 \nu^{2}}{8192}-\frac{625}{131072}\right) Q^{8} .
\end{aligned}
$$


Open Access. This article is distributed under the terms of the Creative Commons Attribution License (CC-BY 4.0), which permits any use, distribution and reproduction in any medium, provided the original author(s) and source are credited.

\section{References}

[1] P.C. Argyres and M.R. Douglas, New phenomena in SU(3) supersymmetric gauge theory, Nucl. Phys. B 448 (1995) 93 [hep-th/9505062] [INSPIRE].

[2] P.C. Argyres, M.R. Plesser, N. Seiberg and E. Witten, New $\mathcal{N}=2$ superconformal field theories in four-dimensions, Nucl. Phys. B 461 (1996) 71 [hep-th/9511154] [INSPIRE].

[3] T. Eguchi, K. Hori, K. Ito and S.-K. Yang, Study of $\mathcal{N}=2$ superconformal field theories in four-dimensions, Nucl. Phys. B 471 (1996) 430 [hep-th/9603002] [INSPIRE].

[4] K. Maruyoshi and J. Song, Enhancement of Supersymmetry via Renormalization Group Flow and the Superconformal Index, Phys. Rev. Lett. 118 (2017) 151602 [arXiv:1606.05632] [INSPIRE].

[5] K. Maruyoshi and J. Song, $\mathcal{N}=1$ deformations and $R G$ flows of $\mathcal{N}=2$ SCFTs, JHEP 02 (2017) 075 [arXiv: 1607.04281] [INSPIRE].

[6] P. Agarwal, K. Maruyoshi and J. Song, $\mathcal{N}=1$ Deformations and $R G$ flows of $\mathcal{N}=2$ SCFTs, part II: non-principal deformations, JHEP 12 (2016) 103 [arXiv:1610.05311] [INSPIRE].

[7] K. Maruyoshi, E. Nardoni and J. Song, Landscape of Simple Superconformal Field Theories in 4d, Phys. Rev. Lett. 122 (2019) 121601 [arXiv:1806.08353] [INSPIRE].

[8] P. Agarwal, A. Sciarappa and J. Song, $\mathcal{N}=1$ Lagrangians for generalized Argyres-Douglas theories, JHEP 10 (2017) 211 [arXiv:1707.04751] [INSPIRE].

[9] S. Benvenuti and S. Giacomelli, Abelianization and sequential confinement in $2+1$ dimensions, JHEP 10 (2017) 173 [arXiv:1706.04949] [INSPIRE].

[10] S. Benvenuti and S. Giacomelli, Lagrangians for generalized Argyres-Douglas theories, JHEP 10 (2017) 106 [arXiv: 1707.05113] [INSPIRE].

[11] S. Giacomelli, RG flows with supersymmetry enhancement and geometric engineering, JHEP 06 (2018) 156 [arXiv:1710.06469] [INSPIRE].

[12] S. Giacomelli, Infrared enhancement of supersymmetry in four dimensions, JHEP 10 (2018) 041 [arXiv: 1808.00592] [INSPIRE].

[13] F. Carta, S. Giacomelli and R. Savelli, SUSY enhancement from T-branes, JHEP 12 (2018) 127 [arXiv: 1809.04906] [INSPIRE].

[14] M.-x. Huang and A. Klemm, Holomorphicity and Modularity in Seiberg-Witten Theories with Matter, JHEP 07 (2010) 083 [arXiv:0902.1325] [INSPIRE].

[15] G. Bonelli, O. Lisovyy, K. Maruyoshi, A. Sciarappa and A. Tanzini, On Painlevé/gauge theory correspondence, arXiv: 1612.06235 [INSPIRE].

[16] O. Gamayun, N. Iorgov and O. Lisovyy, Conformal field theory of Painlevé VI, JHEP 10 (2012) 038 [Erratum ibid. 10 (2012) 183] [arXiv:1207.0787] [INSPIRE].

[17] O. Gamayun, N. Iorgov and O. Lisovyy, How instanton combinatorics solves Painlevé VI, V and IIIs, J. Phys. A 46 (2013) 335203 [arXiv:1302.1832] [INSPIRE]. 
[18] N. Iorgov, O. Lisovyy and J. Teschner, Isomonodromic tau-functions from Liouville conformal blocks, Commun. Math. Phys. 336 (2015) 671 [arXiv:1401.6104] [INSPIRE].

[19] M.A. Bershtein and A.I. Shchechkin, Bilinear equations on Painlevé $\tau$ functions from CFT, Commun. Math. Phys. 339 (2015) 1021 [arXiv: 1406.3008] [INSPIRE].

[20] P. Gavrylenko and O. Lisovyy, Fredholm Determinant and Nekrasov Sum Representations of Isomonodromic Tau Functions, Commun. Math. Phys. 363 (2018) 1 [arXiv:1608.00958] [INSPIRE].

[21] K. Sakai, BPS index and $4 d \mathcal{N}=2$ superconformal field theories, JHEP 07 (2016) 046 [arXiv: 1603.09108] [INSPIRE].

[22] K. Ito and T. Okubo, Quantum periods for $\mathcal{N}=2 \mathrm{SU}(2) S Q C D$ around the superconformal point, Nucl. Phys. B 934 (2018) 356 [arXiv:1804.04815] [INSPIRE].

[23] K. Ito, S. Koizumi and T. Okubo, Quantum Seiberg-Witten curve and Universality in Argyres-Douglas theories, Phys. Lett. B 792 (2019) 29 [arXiv:1903.00168] [INSPIRE].

[24] H. Nagoya, Irregular conformal blocks, with an application to the fifth and fourth Painlevé equations, J. Math. Phys. 56 (2015) 123505 [arXiv: 1505.02398] [INSPIRE].

[25] H. Nagoya, Conformal blocks and Painlevé functions, arXiv:1611.08971 [INSPIRE].

[26] H. Nagoya, Remarks on irregular conformal blocks and Painlevé III and II tau functions, arXiv: 1804.04782 [INSPIRE].

[27] O. Lisovyy, H. Nagoya and J. Roussillon, Irregular conformal blocks and connection formulae for Painlevé V functions, J. Math. Phys. 59 (2018) 091409 [arXiv: 1806.08344] [InSPIRE].

[28] L.F. Alday, D. Gaiotto and Y. Tachikawa, Liouville Correlation Functions from Four-dimensional Gauge Theories, Lett. Math. Phys. 91 (2010) 167 [arXiv:0906.3219] [INSPIRE].

[29] D. Gaiotto, Asymptotically free $\mathcal{N}=2$ theories and irregular conformal blocks, J. Phys. Conf. Ser. 462 (2013) 012014 [arXiv:0908.0307] [INSPIRE].

[30] G. Bonelli, K. Maruyoshi and A. Tanzini, Wild Quiver Gauge Theories, JHEP 02 (2012) 031 [arXiv:1112.1691] [INSPIRE].

[31] D. Gaiotto and J. Teschner, Irregular singularities in Liouville theory and Argyres-Douglas type gauge theories, I, JHEP 12 (2012) 050 [arXiv:1203.1052] [INSPIRE].

[32] H. Kanno, K. Maruyoshi, S. Shiba and M. Taki, $W_{3}$ irregular states and isolated $\mathcal{N}=2$ superconformal field theories, JHEP 03 (2013) 147 [arXiv: 1301.0721] [INSPIRE].

[33] Y. Matsuo, C. Rim and H. Zhang, Construction of Gaiotto states with fundamental multiplets through Degenerate DAHA, JHEP 09 (2014) 028 [arXiv:1405.3141] [INSPIRE].

[34] D. Polyakov and C. Rim, Irregular Vertex Operators for Irregular Conformal Blocks, Phys. Rev. D 93 (2016) 106002 [arXiv:1601.07756] [InSPIRE].

[35] D. Polyakov and C. Rim, Vertex Operators for Irregular Conformal Blocks: Supersymmetric Case, Phys. Rev. D 94 (2016) 086011 [arXiv:1604.08741] [InSPIRE].

[36] C. Rim and H. Zhang, Nekrasov and Argyres-Douglas theories in spherical Hecke algebra representation, Nucl. Phys. B 919 (2017) 182 [arXiv: 1608.05027] [INSPIRE].

[37] A. Grassi and J. Gu, Argyres-Douglas theories, Painlevé II and quantum mechanics, JHEP 02 (2019) 060 [arXiv: 1803.02320] [INSPIRE]. 
[38] H. Itoyama, T. Oota and K. Yano, Discrete Painleve system and the double scaling limit of the matrix model for irregular conformal block and gauge theory, Phys. Lett. B 789 (2019) 605 [arXiv: 1805. 05057] [INSPIRE].

[39] H. Itoyama, T. Oota and K. Yano, Discrete Painleve system for the partition function of $N_{f}=2 \mathrm{SU}(2)$ supersymmetric gauge theory and its double scaling limit, arXiv:1812.00811 [INSPIRE].

[40] H. Itoyama, K. Maruyoshi and T. Oota, The Quiver Matrix Model and 2d-4d Conformal Connection, Prog. Theor. Phys. 123 (2010) 957 [arXiv:0911.4244] [INSPIRE].

[41] T. Eguchi and K. Maruyoshi, Penner Type Matrix Model and Seiberg-Witten Theory, JHEP 02 (2010) 022 [arXiv:0911.4797] [INSPIRE].

[42] R. Schiappa and N. Wyllard, An $A_{R}$ threesome: Matrix models, $2 d$ CFTs and $4 d \mathcal{N}=2$ gauge theories, J. Math. Phys. 51 (2010) 082304 [arXiv:0911.5337] [INSPIRE].

[43] A. Mironov, A. Morozov and S. Shakirov, Matrix Model Conjecture for Exact BS Periods and Nekrasov Functions, JHEP 02 (2010) 030 [arXiv:0911.5721] [INSPIRE].

[44] M. Fujita, Y. Hatsuda and T.-S. Tai, Genus-one correction to asymptotically free Seiberg-Witten prepotential from Dijkgraaf-Vafa matrix model, JHEP 03 (2010) 046 [arXiv: 0912.2988] [INSPIRE].

[45] H. Itoyama and T. Oota, Method of Generating q-Expansion Coefficients for Conformal Block and $\mathcal{N}=2$ Nekrasov Function by beta-Deformed Matrix Model, Nucl. Phys. B 838 (2010) 298 [arXiv: 1003.2929] [INSPIRE].

[46] A. Mironov, A. Morozov and A. Morozov, Conformal blocks and generalized Selberg integrals, Nucl. Phys. B 843 (2011) 534 [arXiv: 1003.5752] [INSPIRE].

[47] A. Morozov and S. Shakirov, The matrix model version of AGT conjecture and CIV-DV prepotential, JHEP 08 (2010) 066 [arXiv:1004.2917] [INSPIRE].

[48] T. Eguchi and K. Maruyoshi, Seiberg-Witten theory, matrix model and AGT relation, JHEP 07 (2010) 081 [arXiv: 1006.0828] [INSPIRE].

[49] H. Itoyama, T. Oota and N. Yonezawa, Massive Scaling Limit of beta-Deformed Matrix Model of Selberg Type, Phys. Rev. D 82 (2010) 085031 [arXiv:1008.1861] [InSPIRE].

[50] K. Maruyoshi and F. Yagi, Seiberg-Witten curve via generalized matrix model, JHEP 01 (2011) 042 [arXiv: 1009.5553] [INSPIRE].

[51] G. Bonelli, K. Maruyoshi, A. Tanzini and F. Yagi, Generalized matrix models and AGT correspondence at all genera, JHEP 07 (2011) 055 [arXiv:1011.5417] [INSPIRE].

[52] H. Itoyama and N. Yonezawa, $\epsilon$-Corrected Seiberg-Witten Prepotential Obtained From Half Genus Expansion in beta-Deformed Matrix Model, Int. J. Mod. Phys. A 26 (2011) 3439 [arXiv:1104.2738] [INSPIRE].

[53] T. Nishinaka and C. Rim, $\beta$-Deformed Matrix Model and Nekrasov Partition Function, JHEP 02 (2012) 114 [arXiv:1112.3545] [inSPIRE].

[54] D. Galakhov, A. Mironov and A. Morozov, S-duality as a beta-deformed Fourier transform, JHEP 08 (2012) 067 [arXiv: 1205.4998] [InSPIRE].

[55] J.-E. Bourgine, Large $N$ limit of beta-ensembles and deformed Seiberg-Witten relations, JHEP 08 (2012) 046 [arXiv: 1206.1696] [InSPIRE]. 
[56] T. Nishinaka and C. Rim, Matrix models for irregular conformal blocks and Argyres-Douglas theories, JHEP 10 (2012) 138 [arXiv:1207.4480] [INSPIRE].

[57] C. Rim, Irregular conformal block and its matrix model, arXiv:1210.7925 [INSPIRE].

[58] S.-K. Choi and C. Rim, Parametric dependence of irregular conformal block, JHEP 04 (2014) 106 [arXiv:1312.5535] [INSPIRE].

[59] H. Itoyama, T. Oota and R. Yoshioka, 2d-4d Connection between $q$-Virasoro/W Block at Root of Unity Limit and Instanton Partition Function on ALE Space, Nucl. Phys. B 877 (2013) 506 [arXiv: 1308.2068] [INSPIRE].

[60] H. Itoyama, T. Oota and R. Yoshioka, $q$-Virasoro/W Algebra at Root of Unity and Parafermions, Nucl. Phys. B 889 (2014) 25 [arXiv:1408.4216] [InSPIRE].

[61] S.K. Choi, C. Rim and H. Zhang, Virasoro irregular conformal block and beta deformed random matrix model, Phys. Lett. B $\mathbf{7 4 2}$ (2015) 50 [arXiv:1411.4453] [INSPIRE].

[62] C. Rim and H. Zhang, Classical Virasoro irregular conformal block, JHEP 07 (2015) 163 [arXiv: 1504.07910] [INSPIRE].

[63] S.K. Choi and C. Rim, Irregular matrix model with $\mathcal{W}$ symmetry, J. Phys. A 49 (2016) 075201 [arXiv: 1506.02421] [INSPIRE].

[64] C. Rim and H. Zhang, Classical Virasoro irregular conformal block II, JHEP 09 (2015) 097 [arXiv: 1506.03561] [INSPIRE].

[65] S.K. Choi, C. Rim and H. Zhang, Irregular conformal block, spectral curve and flow equations, JHEP 03 (2016) 118 [arXiv:1510.09060] [INSPIRE].

[66] D. Polyakov and C. Rim, Super-spectral curve of irregular conformal blocks, JHEP 12 (2016) 004 [arXiv: 1608.04921] [INSPIRE].

[67] N.A. Nekrasov, Seiberg-Witten prepotential from instanton counting, Adv. Theor. Math. Phys. 7 (2003) 831 [hep-th/0206161] [INSPIRE].

[68] D. Xie, General Argyres-Douglas Theory, JHEP 01 (2013) 100 [arXiv:1204.2270] [InSPIRE].

[69] S. Cecotti, A. Neitzke and C. Vafa, R-Twisting and 4d/2d Correspondences, arXiv: 1006.3435 [INSPIRE].

[70] E. Witten, Solutions of four-dimensional field theories via M-theory, Nucl. Phys. B 500 (1997) 3 [hep-th/9703166] [INSPIRE].

[71] D. Gaiotto, $\mathcal{N}=2$ dualities, JHEP 08 (2012) 034 [arXiv:0904.2715] [INSPIRE].

[72] D. Gaiotto, G.W. Moore and A. Neitzke, Wall-crossing, Hitchin Systems and the WKB Approximation, arXiv:0907.3987 [INSPIRE].

[73] K. Ito, S. Kanno and T. Okubo, Quantum periods and prepotential in $\mathcal{N}=2 \mathrm{SU}(2) S Q C D$, JHEP 08 (2017) 065 [arXiv: 1705.09120] [INSPIRE]. 